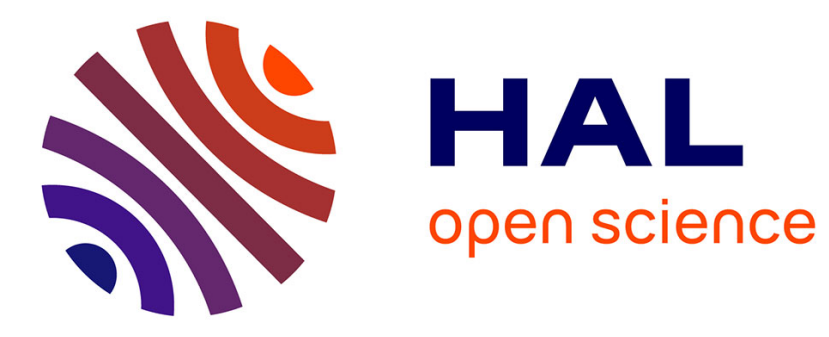

\title{
On importance indices in multicriteria decision making
}

\author{
Michel Grabisch, Christophe Labreuche, Mustapha Ridaoui
}

\section{To cite this version:}

Michel Grabisch, Christophe Labreuche, Mustapha Ridaoui. On importance indices in multicriteria decision making. European Journal of Operational Research, 2019, 277 (1), pp.269-283. 10.1016/j.ejor.2019.02.035 . halshs-02380863

\section{HAL Id: halshs-02380863 \\ https://shs.hal.science/halshs-02380863}

Submitted on 26 Nov 2019

HAL is a multi-disciplinary open access archive for the deposit and dissemination of scientific research documents, whether they are published or not. The documents may come from teaching and research institutions in France or abroad, or from public or private research centers.
L'archive ouverte pluridisciplinaire HAL, est destinée au dépôt et à la diffusion de documents scientifiques de niveau recherche, publiés ou non, émanant des établissements d'enseignement et de recherche français ou étrangers, des laboratoires publics ou privés. 


\title{
On importance indices in multicriteria decision making*
}

\author{
Michel GRABISCH${ }^{1}$, Christophe LABREUCHE ${ }^{2}$ and Mustapha RIDAOUI ${ }^{1 \dagger}$ \\ ${ }^{1}$ Paris School of Economics, University of Paris I \\ 106-112, Bd de l'Hôpital, 75013 Paris, France \\ michel.grabisch@univ-paris1.fr, mustapha.ridaoui@univ-paris1.fr \\ 2 Thales Research and Technology \\ 1, Avenue Augustin Fresnel, 91767 Palaiseau, France \\ christophe.labreuche@thalesgroup.com
}

\begin{abstract}
We address the problem of how to define an importance index in multicriteria decision problems, when a numerical representation of preferences is given. We make no restrictive assumption on the model, which could have discrete or continuous attributes, and in particular, it is not assumed that the model is monotonically increasing or decreasing with respect to the attributes. Our analysis first considers discrete models, which are seen to be equivalent to multichoice games. We propose essentially two importance indices, namely the signed importance index and the absolute importance index, both based on the average variation of the value of the model induced by a given attribute. We provide several axiomatizations for these importance indices, extend them to the continuous case, and finally illustrate them with examples: classical simple models and an example of discomfort evaluation based on real data.
\end{abstract}

Keywords: Multiple criteria analysis, Multichoice game, Shapley value, Choquet integral.

\section{Introduction}

The notion of importance of attributes or criteria in multicriteria decision aiding (MCDA) has always been central in the modelling and analysis of preferences. It is crucial for practitioners for many reasons. Once the model is learnt, the user needs to check the validity of the model, in particular, by checking whether the mean importance of criteria fits with his feeling. In the opposite case, he shall revise his preferences. If some criteria/attribute turn out to have a very small importance, they can be discarded in an approximate and simplified model. On the other hand, if the user wishes to have a synthetic explanation of the model, focusing on the few criteria/attributes having the largest mean importance provides a simple explanation of the model. Finally, the user is often asking for explanations of decisions made by the model. A possible approach is to compute the importance of each criterion for a particular decision (Labreuche and Fossier, 2018). It is instructive to compare this "local" importance with the mean importance. For instance, a mean importance higher than the local importance implies that the corresponding criterion has been weakened by other ones for that particular decision.

What does it mean that a criterion is important and how can it be quantified under the form of an importance index? This question can have a simple answer in simple models based

\footnotetext{
* The paper is a follow-up of two papers of the authors presented at ECSQARU 2017 and ADT 2017 (Ridaoui et al., 2017a,b)

${ }^{\dagger}$ Corresponding author.
} 
on aggregation of scores on individual criteria, where the aggregation is done by the weighted arithmetic mean. Then it is tempting to say that the weights used in the aggregation may be considered as the importance indices of the criteria. This intuition reveals to be correct only under certain circumstances, namely when the scores to be aggregated are calibrated on the same scale and that no other device enters the decision process, like vetos, thresholds, etc.

Within the above framework, when more sophisticated aggregation models are used, like the Choquet integral (Choquet, 1953; Grabisch, 1996; Grabisch and Labreuche, 2010), the Shapley value, a concept borrowed from game theory, is often used. The reason is that the Choquet integral is a generalization of the weighted arithmetic mean, whose "weights" are replaced by a set function $v$ called capacity or fuzzy measure. Capacities can be seen as particular coalitional (TU) games, which are functions $v$ assigning to coalitions of players their benefit or worth. The Shapley value (Shapley, 1953) is a vector assigning to each player its "final" benefit or worth, which is a sharing of the benefit $v(N)$ achieved by the set $N$ of all players. Then, in the MCDA context, $N$ is taken as the set of criteria, and $v(S)$ for any $S \subseteq N$, is intuitively the "weight of importance" of the group of criteria $S$. Considering $v(N)$ as the total importance of all criteria, the Shapley value then expresses the individual importance of criteria.

For other sophisticated models or not necessarily of the aggregation type, like the GAI (Generalized Additive Independence) model of (Fishburn, 1967), we are not aware of any general definition of an importance index. This paper precisely aims at filling this gap.

Our approach is quantitative, as our aim is to define a numerical importance index, and therefore our starting point will be a numerical representation of the preferences, rather than the preferences itself. The strong point of our approach is that no specific assumption is needed on the numerical representation. In particular, we can deal with continuous or discrete-valued attributes, although most of our effort will bear on the discrete case, the continuous case being obtained by interpolation. Also, we do not assume monotonicity, that is, the satisfaction of the decision maker does not necessarily increase nor decrease with the values of the attributes.

Basically, we propose two families of importance indices, plus a natural generalization of the second one. The first one considers that the importance index of attribute $i$ is a (weighted) average of the variation of the model along the $i$ axis. This idea comes from the field of aggregation functions, and it is known from Marichal (1998) that applied to the Choquet integral, it yields the Shapley value. We show, however, that this approach is meaningful only if the model is monotone with respect to (w.r.t.) the attributes, otherwise the average variation along an axis could be close to zero, simply because positive variations cancel negative ones. This is why we propose a second approach, taking the cumulation of absolute variations rather than the average of variations. This leads to the family of absolute importance indices, contrasting with the former one called signed importance index. Realizing that taking the sum of absolute values amounts to consider the $L_{1}$ norm, a third family, called norm-based importance indices, is proposed where the amount of variation is computed by a norm. This family generalizes the absolute importance index.

Our work makes also a contribution to the theory of multichoice games (Hsiao and Raghavan, 1993), by providing alternative definitions of Shapley-like values for multichoice games. Indeed, in the discrete case, a numerical model of preference can be seen as a multichoice game (see, e.g., Grabisch and Labreuche (2018); Labreuche and Grabisch (2018)). Our approach is axiomatic, so as to ensure rational and clear properties for the defined indices. Also, it shows clearly the difference with the values proposed in the literature of game theory. These values satisfy the axiom of efficiency, which is rooted in game theory, but, as we will explain below, it has no reason to hold in a MCDA context. This is why these values cannot be used as importance indices, which motivates our study. Lastly, we mention that the usage of importance indices is not limited to the field of MCDA: having an information on which variables are the most 
influential on a model $U$ is very useful in Experimental Design, and also for the interpretation of Classification or Machine Learning models (Datta et al.; Lundberg and Lee, 2017). These two references use Shapley or Banzhaf indices to interpret a classification model. The use of such indices has recently become popular in AI - under the domain of Explainable AI (XAI).

The paper is organized as follows. Section 2 introduces the framework, notation and the necessary background. Section 3 is a discussion on how to define importance indices, introducing our three families. The related literature is given in Section 4. The main part of the paper consists in Sections 5 and 6, giving the axiomatizations in the discrete case (hence equivalent to a multichoice game) of the signed and the absolute importance indices (another axiomatization of both indices is also presented in Section 10). Section 7 gives an axiomatization of the family of norm-based indices. The usage of the signed and absolute importance indices are illustrated on an example in Section 8. Section 9 addresses the continuous case. It shows that if interpolation is done by the Choquet integral, then the previous definitions of signed and absolute importance indices are still valid for the continuous case. The appendices contain the proofs of the results and show the logical independence of the axioms used to characterize the signed importance indices.

To summarize, the highlights of our approach are:

(i) It is crucial in applications to provide the mean importance of criteria/attributes, in MCDA but also in other fields, especially Machine Learning.

(ii) The current proposals for defining criteria importance are model specific. We provide model-agnostic importance indices without specific assumptions (monotonicity, continuous/discrete, etc.).

(iii) Two complementary importance indices are proposed. The signed index indicates the overall trend of the model w.r.t. an attribute (either increasing or decreasing). The absolute index measures the amount of variation of the model induced by an attribute regardless of its monotonicity.

(iv) The proposed indices contribute to the theory of multichoice games, by providing alternative definitions of the Shapley value.

\section{The framework}

Throughout the paper, the cardinality of sets will be denoted by corresponding lower case letters, e.g., $|N|=n,|S|=s$, etc. For simplicity, singletons $\{i\}$ are often denoted by $i$.

We consider a multicriteria decision aiding (MCDA) problem with $n$ attributes $X_{1}, \ldots, X_{n}$. We denote by $N=\{1, \ldots, n\}$ the index set of attributes. The set of potential alternatives is therefore the Cartesian product $X=X_{1} \times \cdots \times X_{n}$.

There is no restriction on the $X_{i}$ 's, i.e., they may be continuous $\left(X_{i}=\mathbb{R}\right.$ or is an interval of $\mathbb{R}$ ) or discrete sets, however, in the former case, we select in each attribute $X_{i}$ a finite set of values $a_{0}^{i}, \ldots, a_{k_{i}}^{i}$, called levels, $\left(\forall i \in N, k_{i} \in \mathbb{N}\right.$, such that $\left.k_{i} \geq 1\right)$. There must be for each attribute at least two levels (these would be the upper and lower bounds of $X_{i}$ when it is an interval of $\mathbb{R}$ ). For the most part of our development (definition and axiomatization of some importance indices), we consider only these levels, so that all is working as if the attributes were discrete. We address the continuous case in Section 9.

The precise value of the chosen levels (or values of the discrete attributes) $a_{j}^{i}$ playing no role in our development (except in Section 9), we refer to them by simply giving their subindex, i.e., alternative $\left(a_{j_{1}}^{1}, a_{j_{2}}^{2}, \ldots, a_{j_{n}}^{n}\right)$ is represented by $\left(j_{1}, j_{2}, \ldots, j_{n}\right)$. This amounts to considering 
alternatives as elements of the Cartesian product $L:=L_{1} \times \cdots \times L_{n}$, with $L_{i}=\left\{0,1, \ldots, k_{i}\right\}$. Also, we introduce $\Gamma(L)=\left\{0, k_{1}\right\} \times \cdots \times\left\{0, k_{n}\right\}$, the set of "vertices" of $L$. Although a discrete attribute $X_{i}$ is not necessarily ordered, our notation using $L_{i}$ automatically induces an implicit ordering on the values of the attribute: $a_{j}^{i} \leq a_{k}^{i}$ iff $j \leq k$. This also induces a partial order on $L$ by taking the product order: for $x, y \in L, x \leq y$ if $x_{i} \leq y_{i}$ for all $i=1, \ldots, n$. We write $x \lessgtr y$ if $x \leq y$ and $x \neq y$ for every $x, y \in L$.

As customary in decision theory, for any $x \in X$ (or $x \in L$ ) and $S \subseteq N, x_{S}$ denotes the restriction of $x$ to the coordinates in $S$, while $x_{-S}$ denotes the restriction to the coordinates in $N \backslash S$. Accordingly, we use $L_{S}, X_{S}, L_{-S}, X_{-S}$ for the restricted Cartesian products of attributes. Hence, with $x_{-i} \in L_{-i}$ and $\ell \in L_{i},\left(x_{-i}, \ell_{i}\right)$ is an element of $L$ whose coordinate $i$ is $\ell$ and $x_{-i}$ is the vector of the other coordinates. For an alternative $x \in L$, and $i \in N$ such that $x_{i}+1 \leq k_{i}$ $x+1_{i}$ denotes the vector $\left(x_{-i}, x_{i}+1\right)$. As far as no confusion arises, $\left(x_{-i},\left(k_{i}\right)_{i}\right)$ is simply denoted by $\left(x_{-i}, k_{i}\right)$. The vector $0_{N}=(0, \ldots, 0)$ is the null alternative of $L$, and $k_{N}=\left(k_{1}, \ldots, k_{n}\right)$ is the top element of $L$. For each $x \in L$, we denote by $\Sigma(x)=\left\{i \in N \mid x_{i}>0\right\}$ the support of $x$, and by $\mathrm{K}(x)=\left\{i \in N \mid x_{i}=k_{i}\right\}$ the kernel of $x$. Their cardinalities are respectively denoted by $\sigma(x)$ and $\kappa(x)$.

We suppose that the preferences of the decision maker over alternatives in $L$ can be represented numerically by a value function $v: L \rightarrow \mathbb{R}$ (Note: It is out of the scope of this paper to say how $v$ can be obtained; depending on its chosen form, many classical methods in MCDA and MAUT can be applied for this). We suppose that $v\left(0_{N}\right)=0$, which is no restriction since most of the time numerical representation of preferences are given up to a positive affine transformation.

Apart this, we make no special assumption on $v$ : the value function could be additive, or be of more sophisticated form (e.g., Generalized Additive Independence (GAI) model, etc.), anyway, we use in the sequel only its numerical values $v(x)$ for each $x \in L$.

In particular, the classical property of (increasing) monotonicity, which says that

$$
x \leq y \Rightarrow v(x) \leq v(y),
$$

or its converse (decreasing monotonicity) or any mixed version, are not assumed here. Monotonicity means that the higher (or the lower) the value of the attributes, the better the alternative. If this holds in many practical examples (cost, production time, benefit, etc.), it is not true in general that maximizing or minizing attribute values maximizes the satisfaction of the decision maker: it may be the case that the ideal value of the attribute is not one of the bounds. The following example shows this.

Example 1 (Employee working conditions). We consider a function $v$ measuring the overall degree of well-being at work. A high degree indicates that the employee is comfortably seated with ideal conditions allowing a good productivity. To keep it simple, we consider three attributes: the temperature $X_{1}$ of the room, the light intensity $X_{2}$ (for artificial lightening), and the level of comfort $X_{3}$ of the chair. One can readily see that the optimal temperature is not reached for extreme values. The ideal temperature is more likely to be optimal around 22 degree Celsius. Likewise, the ideal light intensity should not be too strong for the employee's eyes. Finally the level of comfort of the chair that is best for the standpoint of his productivity is neither too low (which can generate pain in the back of the employee) nor too high (the employee might fall asleep). To sum-up, for each attribute, there exists an optimal value $\widehat{\ell}_{i} \in L_{i}$ such that $v$ is increasing in $x_{i}$ below $\widehat{\ell}_{i}$, and then decreasing in $x_{i}$ above $\widehat{\ell}_{i}$.

Observe that in Example 1, the preference is single-peaked: on each attribute, the function $v$ is increasing till an optimal value is reached, and then decreasing. A drastic example of 
single-peaked value function is the $\delta_{x}$ Dirac function, defined by, for any $x \in L \backslash\left\{0_{N}\right\}$,

$$
\delta_{x}(y)= \begin{cases}1, & \text { if } y=x \\ 0, & \text { otherwise }\end{cases}
$$

It is worth noting that this general framework permits to recover some classical concepts. When $k_{1}=\ldots=k_{n}=1, v$ is a pseudo-Boolean function (Crama and Hammer, 2011; Hammer and Rudeanu, 1968) $v:\{0,1\}^{N} \rightarrow \mathbb{R}$ vanishing at $0_{N}$. Through the correspondence between a set $S$ and its characteristic function $1_{S}$, it can be put in the form of a function $\mu: 2^{N} \rightarrow \mathbb{R}$, which is a TU-game in cooperative game theory (see, e.g., Peleg and Sudhölter (2003); Peters (2008)). When monotonicity (1) is enforced, we obtain capacities introduced by Choquet (1953). When $k_{i} \geq 1$ for each $i \in N$, multichoice games, introduced by Hsiao and Raghavan (1993), are recovered. Monotone multichoice games, i.e., satisfying (1), were introduced by Grabisch and Labreuche (2003) under the name of $k_{N}$-ary capacities.

It turns out that in any case, our numerical representation $v$ on $L$ can be seen as a multichoice game. We will therefore work on the set of multichoice games on $L$, which we denote by $\mathcal{G}(L)$. In addition, $\mathcal{G}_{M}(L)$ is the set of monotone multichoice games.

We finish this section by introducing some technical notions on multichoice games. The derivative of $v$ w.r.t. an attribute $i$ at point $x \in L$ such that $x_{i}<k_{i}$ is defined by

$$
\Delta_{i} v(x)=v\left(x+1_{i}\right)-v(x) .
$$

The set $\mathcal{G}(L)$ of multichoice games is a vector space of dimension $k_{1} k_{2} \cdots k_{n}-1$. A trivial basis of $\mathcal{G}(L)$ is the set $\left\{\delta_{x}\right\}_{x \in L \backslash\left\{0_{N}\right\}}$ introduced above. Another one is the basis of unanimity games $u_{x}$, which are defined for any $x \in L \backslash\left\{0_{N}\right\}$ by

$$
u_{x}(y)= \begin{cases}1, & \text { if } y \geq x \\ 0, & \text { otherwise }\end{cases}
$$

Then, for any game $v \in \mathcal{G}$,

$$
v=\sum_{x \in L \backslash\left\{0_{N}\right\}} m^{v}(x) u_{x} .
$$

The coordinates $m^{v}(x), x \in L \backslash\left\{0_{N}\right\}$, of a game $v$ are known to be the Möbius transform of $v$ (Rota, 1964), which can be seen as a function $m^{v}: L \rightarrow \mathbb{R}$ with $m^{v}\left(0_{N}\right):=0$. It is useful to consider $\mathcal{G}_{+}(L)$ the set of games whose Möbius transform is nonnegative, and to note that $\mathcal{G}_{+}(L) \subseteq \mathcal{G}_{M}(L)$ is a cone. It is easy to see, as for classical games, that any multichoice game $v \in \mathcal{G}(L)$ can be uniquely decomposed into

$$
v=v^{+}-v^{-}
$$

with $v^{+}, v^{-} \in \mathcal{G}_{+}(L)\left(v^{+}, v^{-}\right.$simply correspond respectively to the nonnegative coefficients and negative coefficients of the Möbius transform of $v$ ).

\section{How to define importance indices}

Our aim is to define an importance index in this general framework, where no special assumption is made on the model, numerically represented by $v$, apart that for the moment, $v$ is defined on discrete attributes.

The value function $v$ expresses the intensity of satisfaction of the decision maker for the different alternatives in $L$, which are multidimensional vectors $x=\left(x_{1}, \ldots, x_{n}\right)$. In this sense, 
$v$ can be seen as an aggregation function, yielding a single real number from $n$ inputs. The importance index of an attribute should reflect to which extent the attribute influences the output value. As this influence may depend on the value of the other attributes, a kind of average influence should be taken. As the output value is a real number, influence is visible only through a change or variation of this value. Hence, the importance of an attribute is related to the amount of variation this attribute can produce on $v$. This view is in fact the one taken in the domain of aggregation functions $F: I^{n} \rightarrow I$, where $I$ is some real interval (see, e.g., Grabisch et al. (2009)[§10.3]). The importance index of attribute (dimension) $i$ is defined by, assuming $I=[a, b]$,

$$
\phi_{i}(F)=\frac{1}{(b-a)^{n}} \int_{[a, b]^{n}} \frac{\Delta_{i} F(x)}{b-a} d x=\frac{1}{(b-a)^{n}} \int_{[a, b]^{n}} \frac{\partial F(x)}{\partial x_{i}} d x
$$

where $\Delta_{i} F(x)=F\left(b_{i}, x_{-i}\right)-F\left(a_{i}, x_{-i}\right), x \in I^{n}$. As aggregation functions are always nondecreasing in each argument, $\Delta_{i} F(x)$ is the total variation on dimension $i$ at $x$, while the second equality makes apparent that the importance index of $i$ is an average of the local variation due to $i$.

We apply the same idea to the value function $v$, although $v$ is not supposed to be monotone and is defined on a discrete domain. A general expression for the importance index of attribute $i$ w.r.t. $v$ would be, using the (discrete) derivative (3):

$$
\phi_{i}(v)=\sum_{\substack{x \in L \\ x_{i}<k_{i}}} p_{x}^{i} \Delta_{i} v(x)
$$

where the $p_{x}^{i}$ are real coefficients (weights). If the weights $p_{x}^{i}$ depend only on $p_{x_{-i}}^{i}$, then

$$
\sum_{x_{i}=0}^{k_{i}-1} p_{x_{-i}}^{i} \Delta_{i} v(x)=p_{x_{-i}}^{i}\left(v\left(x_{-i}, k_{i}\right)-v\left(x_{-i}, 0_{i}\right)\right),
$$

which reminds the first equality in (7). We will specify further and axiomatize the family of importance indices defined in (8) in Section 5.

Observe that in case of an aggregation function or a monotone increasing value function $v$, the importance index defined in (7) or (8) is a positive quantity, while it becomes negative when the considered function is monotone decreasing. For this reason, we may call such type of importance index a signed importance index.

Signed importance indices may give counterintuitive results, though. Suppose that the value function $v$ is such that, roughly speaking, there is as much positive variation as negative variation along the $i$ axis. The total variation would be then zero, which seems to indicate that attribute $i$ has no importance, although it does have since it induces some variation of the function $v$. This is exactly the situation given in Example 1: for every attribute, when ranging from lowest value to highest value of the attribute, the variation is first positive and then negative, and we conclude that all attributes have a negligible importance. The extreme example is given by the $\delta_{x}$ function (2), where all attributes have zero importance for all $x$ such that $0<x_{i}<k_{i}$ for all $i \in N$ (provided that the weights have some suitable symmetry, e.g., $p_{x}^{i}$ does not depend on $x_{i}$ ).

A simple way to avoid this drawback is to cumulate the local variations, instead of summing them algebraically; put differently, the sum of the absolute value of the local variations should be taken:

$$
\phi_{i}(v)=\sum_{\substack{x \in L \\ x_{i}<k_{i}}} p_{x}^{i}\left|\Delta_{i} v(x)\right| .
$$


We call this family the absolute importance indices. One may go one step further by remarking that the absolute value is the $L_{1}$ norm, and that any norm can be taken instead:

$$
\phi_{i}(v)=\sum_{\substack{x \in L \\ x_{i}<k_{i}}} p_{x}^{i}\left\|\Delta_{i} v(x)\right\|,
$$

or even in a more compact form, considering norms with weights on coordinates:

$$
\phi_{i}(v)=\left\|\Delta_{i}(v)\right\| \quad(i \in N) .
$$

We may call this the norm-based importance indices. In Section 6, we will axiomatize and further specify the family of absolute importance indices, while Section 7 will address the case of norm-based importance indices.

\section{Related works}

Many models in MCDA are additive and based on the weighted arithmetic mean (AHP, ELECTRE, PROMETHEE, etc.), where the weights of the arithmetic are directly taken as weight of importance of the attributes. As for more sophisticated nonadditive models, the problem has been well studied for models based on the Choquet integral (Grabisch and Labreuche, 2010, 2016), however, as far as we know, there is no specific study on GAI models.

We elaborate on Choquet integral-based models. The most basic model in this category has the form:

$$
U(x)=C_{\mu}\left(u_{1}\left(x_{1}\right), \ldots, u_{n}\left(x_{n}\right)\right)
$$

where $C_{\mu}$ indicates the Choquet integral w.r.t. a capacity $\mu$ on $2^{N}$, and $u_{1}, \ldots, u_{n}$ are marginal utility functions on the different attributes which are commensurable. Recall that a capacity is a monotone game, thus it corresponds to the case $k_{1}=\cdots=k_{n}=1$ of multichoice games. It has been suggested first by Murofushi (1992) and later developed by Grabisch (1996) that the Shapley value (Shapley, 1953), a concept borrowed from cooperative game theory, should be used as importance index for such models.

Generally speaking, a value is a mapping $\phi: \mathcal{G}\left(2^{N}\right) \rightarrow \mathbb{R}^{N}$ assigning a payoff vector to any game $v$. The expression of the Shapley value is given by, for any $v \in \mathcal{G}\left(2^{N}\right)$,

$$
\phi_{i}^{S h}(v)=\sum_{S \subseteq N \backslash i} \frac{(n-s-1) ! s !}{n !}(v(S \cup i)-v(S)), \forall i \in N .
$$

The classical intepretation of this formula in game theory is as follows: the players are ordered and they receive their marginal contribution to the coalition formed by the previous players as their payment. Then an average over all orderings is performed. Through the identity between a set $S$ and its characteristic function $1_{S}$, the comparison of (11) with (8) shows that the Shapley value is a particular signed importance index, with $k_{i}=1(\forall i \in N)$ and the weights given by $p_{S}^{i}=\frac{(n-s-1) ! s !}{n !}$. Remembering that a capacity is monotone, it turns out that the Shapley value is also an absolute importance index.

More interestingly, it is shown in Grabisch et al. (2000) that taking $F$ in (7) as the Choquet integral w.r.t. $\mu$, the importance index $\phi_{i}\left(C_{\mu}\right)$ coincides with $\phi_{i}^{\text {Sh }}(\mu)$, the Shapley value of $\mu$, showing the well-foundedness of the Shapley value as importance index for Choquet integralbased models.

We note that a corresponding result holds for the MLE model (multilinear extension of Owen (1972)) and the Banzhaf value (Banzhaf, 1965) (see, again, Grabisch et al. (2000)). 
The previous paragraph shows the relation between importance indices in MCDA and values (Shapley, Banzhaf) in game theory. As our function $v$ when defined on a discrete domain can be interpreted as a multichoice game, the question arises whether Shapley-like values defined for multichoice games can be used as importance indices. Among the many definitions proposed in the literature, let us mention Hsiao and Raghavan (1993) (historically the first one), van den Nouweland et al. (1995), Klijn et al. (1999), Peters and Zank (2005), Grabisch and Lange (2007), etc. The value of Peters and Zank reads:

$$
\phi_{i}^{\mathrm{PZ}}(v)=\sum_{x_{-i} \in \Gamma\left(L_{-i}\right)} \frac{\left(n-\kappa\left(x_{-i}\right)-1\right) ! \kappa\left(x_{-i}\right) !}{n !}\left(v\left(x_{-i}, k\right)-v\left(x_{-i}, 0\right)\right),
$$

with $k_{i}=k, \forall i \in N$.

Clearly, (12) has the form of a signed importance index, although one can remark that only vertices of $L_{-i}$ are taken in the sum.

All these values, for classical games or multichoice games, are characterized by axioms, among which we always find linearity ( $\phi$ is a linear operator on $\mathcal{G}(L)$ ), a null axiom (a player with zero marginal contribution receives zero), a symmetry (or anonymity) axiom (the numbering of the players has no influence on the payoff) and efficiency (the sum of the payoffs for all players should be equal to $v\left(k_{N}\right)$, where $k_{N}$ is the top element of $\left.L\right)$. While the first three axioms are easily transposable in the MCDA framework, the efficiency axiom is rooted in game theory and there is no convincing interpretation in MCDA. Indeed, efficiency means that the total benefit of the cooperation, represented by $v\left(k_{N}\right)$, should be shared among the players with no waste. In MCDA, the concept of sharing is not natural, and there is no reason why the sum of importance indices should be equal to $v\left(k_{N}\right)$. When $v$ is monotone, then $v\left(k_{N}\right)$ is the evaluation of the best possible alternative, and in this case it may be meaningful to consider the importance index of a criterion as a percentage of the best possible evaluation. However, this interpretation fails when $v$ is not monotone. Especially, in Example 1, the value of $v\left(k_{N}\right)$ should be close to 0, as the highest values of the attributes are not considered as comfortable.

This explains why multichoice game values of the literature cannot be used as importance indices, and this motivates our study. It also explains why it is not enough to specify a general form for the family (signed importance index, absolute importance index) as in (8), (9), but one has to specify the weights, in order that the resulting index has suitable properties. This is why we think that the only way is to proceed axiomatically. The next two sections axiomatize some signed and absolute importance indices, which are meaningful in terms of MCDA.

\section{Axiomatization of signed importance indices}

Let us denote by $\phi: \mathcal{G}(N) \rightarrow \mathbb{R}^{N}$ an importance index, where $\mathcal{G}(N)$ denotes the set of all multichoice games on $N$ (that is, with arbitrary number of levels for each $i \in N$, i.e., with any $L)$. The aim of this section is to give an axiomatization of the family defined by (8), and by imposing additional axioms, to further specify possible sets of weights. The first two axioms we will introduce are close to the classical axioms used by Shapley in his seminal paper (Shapley, 1953).

Linearity axiom $(\mathbf{L}): \phi$ is linear on $\mathcal{G}(L)$, i.e., $\forall v, w \in \mathcal{G}(L), \forall \alpha \in \mathbb{R}$,

$$
\phi(v+\alpha w)=\phi(v)+\alpha \phi(w) .
$$

The linearity axiom means that if we have the preferences $v$ and $w$ of two DMs, and the resulting preference is a linear combination of them (yielding $v=\alpha v+\beta w$ ), then it is equivalent to apply $\phi$ before or after the linear transformation. 
The next axiom is an invariance property. It says that the calculus of the importance index does not depend on the position on the "grid" $L$. For this, we need for convenience to consider that, w.r.t. to some attribute $i$, the grid is "periodized" modulo $k_{i}$, i.e.: $\ldots, k_{i}-2, k_{i}-$ $1,0,1, \ldots, k_{i}-1,0, \ldots$

Invariance axiom (I): Let us consider two functions $v, w \in \mathcal{G}(L)$ such that, for some $i \in N$,

$$
\Delta_{i} v(x)=\Delta_{i} w\left(x-1_{i}\right), \quad x_{-i} \in L_{-i}, x_{i} \in\left\{0, \ldots, k_{i}-1\right\}\left(\bmod k_{i}\right) .
$$

Then,

$$
\phi_{i}(v)=\phi_{i}(w) .
$$

Axiom (I) says that if two games $v, w$ have the same increments along an attribute $i$ up to a shift of one unit on this attribute, the importance of attribute $i$ is the same for $v, w$. Note that for $x_{i}=0$ the condition reads $\Delta_{i} v\left(x_{-i}, 0_{i}\right)=\Delta_{i} w\left(x_{-i},\left(k_{i}-1\right)_{i}\right)$. In contrast to the classical symmetry or anonymity axiom which pertains to the identity of the players/attributes, it is a kind of "geographical" symmetry or homogeneity axiom, saying that in the grid, the computation of the index is done without distortion due to the location in the grid.

Remark 1. It can be seen that this axiom implies the null axiom, which is classical in the axiomatization of the Shapley value. It says that a player $i \in N$ who brings no contribution (i.e., $\mu(S \cup i)=\mu(S), \forall S \subseteq N \backslash\{i\}$ : no variation, in our language) should receive a zero payoff (in our language: has no importance). This definition can be easily extended to multichoice games as follows: $i \in N$ is a null attribute for $v \in \mathcal{G}(L)$ if $v\left(x+1_{i}\right)=v(x), \forall x \in L, x_{i}<k_{i}$. The null axiom $(\mathrm{N})$ says that if $i$ is null for $v$, then $\phi_{i}(v)=0$.

The null axiom is implied by (I): indeed, consider $w=0$ (null game). Then the condition on $\Delta_{i} v$ is equivalent to say that $i$ is a null player. It follows from linearity that $\phi_{i}(w)=0$, hence $\phi_{i}(v)=0$ too.

Proposition 1. Under axioms (L) and (I), $\forall v \in \mathcal{G}(L), \forall i \in N$,

$$
\phi_{i}(v)=\sum_{x_{-i} \in L_{-i}} p_{x_{-i}}^{i}\left(v\left(x_{-i}, k_{i}\right)-v\left(x_{-i}, 0_{i}\right)\right), p_{x_{-i}}^{i} \in \mathbb{R}, \forall x_{-i} \in L_{-i} .
$$

All proofs of the propositions and theorems are given in Appendix A.

Axiom $(\mathbf{I})$ in combination with $(\mathbf{L})$ implies that we only need to look at the variation of $v$ between the extreme values 0 and $k_{i}$. The evaluation on the intermediate elements of $L_{i}$ does not intervene.

We now introduce the symmetry axiom.

Symmetry for Dirac games (SD): Let $x \in L \backslash\left\{0_{N}\right\}$.

- $\forall i, j \in K(x), \phi_{i}\left(\delta_{x}\right)=\phi_{j}\left(\delta_{x}\right)$,

- $\forall i, j \in N \backslash \Sigma(x), \phi_{i}\left(\delta_{x}\right)=\phi_{j}\left(\delta_{x}\right)$.

The symmetry axiom means that for the Dirac game, there is an equal importance for attributes in the kernel, or outside the support of the game.

In game theory, it is common to use an efficiency axiom, meaning that the total sum $\sum_{i \in N} \phi_{i}(v)$ (recall that in this context $\phi_{i}(v)$ is interpreted as a payoff given to $i$ ) should be equal to the total worth or benefit, $v\left(k_{N}\right)$. As explained in Section 4, no such interpretation 
holds in our context, but some substitute to efficiency (normalization or calibration axiom) has to be found in order to determine the weights $p_{x_{-i}}^{i}$ uniquely.

As the signed importance index of attribute $i$ reflects the overall (algebraic) variation of $v$ along this axis, the sum of the importance indices should be made equal to the total (algebraic) variation of $v$, which is naturally defined as the sum of variations of $v$ over all positions $x$ in the grid, where all components of $x$ are increased by one unit (i.e., a move along the main diagonal). Moving along the main diagonal from $x$ measures the effect of all attributes simultaneously and permits to capture correlations between variables.

\section{Efficiency axiom (E):}

$$
\sum_{i \in N} \phi_{i}(v)=\sum_{\substack{x \in L \\ \forall j \in N, x_{j}<k_{j}}}\left(v\left(x+1_{N}\right)-v(x)\right)
$$

Remark 2. Interestingly, although the above equation is clearly in the spirit of variation calculus suitable to our approach, making $k_{i}=1, \forall i \in N$ leads to $\sum_{i \in N} \phi_{i}(v)=v\left(1_{N}\right)-v\left(0_{N}\right)=v\left(1_{N}\right)$, i.e., the classical efficiency axiom. It shows that the usual definition of efficiency for multichoice games is not the only way to generalize the classical definition.

Applied to an arbitrary function $v$, the right handside of equation (14) does not give any peculiar result. Hence, let us take the Dirac function $\delta_{x}$, central in our discussion. We have several cases:

- $\kappa(x) \neq 0$ and $\sigma(x)=n$. Then $x-1_{N} \in L$ because no component of $x$ is equal to 0 , and we have $\delta_{x}(x)-\delta_{x}\left(x-1_{N}\right)=1$. Note that $\delta_{x}\left(y+1_{N}\right)-\delta_{x}(y)=0$ for any $y \neq x-1$ and $y, y+1 \in L$. Therefore,

$$
\sum_{i \in N} \phi_{i}\left(\delta_{x}\right)=1
$$

- $\kappa(x)=0$ and $\sigma(x)<n$. Then $x+1_{N} \in L$ because no component of $x$ is equal to $k_{i}$, and we have $\delta_{x}\left(x+1_{N}\right)-\delta_{x}(x)=-1$. Note that $\delta_{x}\left(y+1_{N}\right)-\delta_{x}(y)=0$ for any other possible $y$, therefore,

$$
\sum_{i \in N} \phi_{i}\left(\delta_{x}\right)=-1
$$

- $\kappa(x) \neq 0$ and $\sigma(x)<n$. There are both components equal to 0 and to $k_{i}$ in $x$. Therefore, neither $x+1_{N}$ nor $x-1_{N}$ belong to $L$, and for any possible $y \in L$ s.t. $y+1 \in L$, we have $\delta_{x}\left(y+1_{N}\right)-\delta_{x}(y)=0$. Therefore,

$$
\sum_{i \in N} \phi_{i}\left(\delta_{x}\right)=0
$$

- $\kappa(x)=0$ and $\sigma(x)=n$. We have $\delta_{x}\left(x+1_{N}\right)-\delta_{x}(x)=-1$ and $\delta_{x}(x)-\delta_{x}\left(x-1_{N}\right)=1$, otherwise the difference is 0 for all other $y$. Therefore,

$$
\sum_{i \in N} \phi_{i}\left(\delta_{x}\right)=0
$$

Theorem 1. Under axioms (L), (I), (SD) and (E), for all $v \in \mathcal{G}(L)$

$$
\phi_{i}(v)=\phi_{i}^{\mathrm{s}}(v):=\sum_{x_{-i} \in L_{-i}} \frac{\left(n-\sigma\left(x_{-i}\right)-1\right) ! \kappa\left(x_{-i}\right) !}{\left(n+\kappa\left(x_{-i}\right)-\sigma\left(x_{-i}\right)\right) !}\left(v\left(x_{-i}, k_{i}\right)-v\left(x_{-i}, 0_{i}\right)\right), \forall i \in N
$$


The theorem shows that the signed importance index for $i$ is a weighted average of the total variation of $v$ along the axis $i$. The weights, which are implied by (E), resemble the weights in the formula of the Shapley value.

To show the independence of the four axioms (L), (I), (SD) and (E), we have slightly weakened the invariance axiom (I) by adding the technical condition $v\left(x+1_{N}\right)-v(x)=$ $w\left(x+1_{N}\right)-w(x)$ for each $x \in L$, such that $x_{i}<k_{i}$, for all $i \in N$ (axiom (I')). The additional technical condition does not make the axiom more attractive nor intuitive.

Theorem 2. Under axioms (L), (I'), (SD) and (E), for all $v \in \mathcal{G}(L)$

$$
\phi_{i}(v)=\phi_{i}^{\mathrm{s}}(v)
$$

The logical independence of axioms (L), (I'), (SD) and (E) is shown in Appendix B.

\section{Axiomatization of absolute importance indices}

We now turn to the axiomatization of the family defined by (9), and of particular instances of it. The major difficulty in axiomatizing (9) is that $\phi$ does not satisfy linearity, and therefore it is not possible to start from the decomposition of games in some basis. Instead, the idea is the following: the expression of $v$ in the basis of unanimity games (5) becomes a conic combination when $v$ is in $\mathcal{G}_{+}(L)$. As any game can be written as the difference of two games in $\mathcal{G}_{+}(L)$ (see (6)), it is then possible to extend this expression to monotone games, for which the absolute value in $\left|v\left(x+1_{i}\right)-v(x)\right|$ is no more necessary.

Conic Combination axiom (CC) : For every $v, w \in \mathcal{G}_{M}(L)$, for every $\alpha \in \mathbb{R}_{+}$,

$$
\phi(v+\alpha w)=\phi(v)+\alpha \phi(w) .
$$

The Conic Combination axiom permits to obtain the following result.

Proposition 2. Under axiom (CC), for all $i \in N$, there exists constants $a_{x}^{i} \in \mathbb{R}$, for all $x \in L$, such that for all $v \in \mathcal{G}_{M}(L)$,

$$
\phi_{i}(v)=\sum_{x \in L} a_{x}^{i} v(x) .
$$

Taking two multichoice games $v$ and $w$ for which the marginal contribution of a player $i$ in a game $v$ is the same or the opposite of that in a game $w$, the average importance of attribute $i$ shall be the same for $v$ and $w$. We propose the following axiom.

Marginal contribution axiom (MC): Let $i \in N$ and $v, w \in \mathcal{G}(L)$ such that

$$
\left|\Delta_{i} v(x)\right|=\left|\Delta_{i} w(x)\right|, \forall x \in L, x_{i}<k_{i} .
$$

Then $\phi_{i}(v)=\phi_{i}(w)$.

These two axioms suffice to characterize the family of absolute importance indices.

Proposition 3. Under axioms (CC) and (MC), there exist real constants $p_{x}^{i}, i \in N, x \in$ $L, x_{i}<k_{i}$, such that for every $v \in \mathcal{G}(L)$,

$$
\phi_{i}(v)=\sum_{\substack{x \in L \\ x_{i}<k_{i}}} p_{x}^{i}\left|\Delta_{i} v(x)\right| .
$$


Remark 3. As it can be seen from (22), the null axiom (N) is implied by (CC) and (MC) (see proof of Proposition 3).

We can now further restrict the family by imposing the invariance axiom that was used in Section 5. We obtain:

Proposition 4. Under axioms (CC), (I) and (MC), for all $v \in \mathcal{G}(L)$

$$
\phi_{i}(v)=\sum_{\substack{x \in L \\ x_{i}<k_{i}}} p_{x_{-i}}^{i}\left|v\left(x+1_{i}\right)-v(x)\right|, \forall i \in N .
$$

Absolute Efficiency (AE): Let $x \in L$ such that $N \backslash \Sigma(x) \neq \varnothing$ and $\mathrm{K}(x) \neq \varnothing$.

$$
\sum_{i \in \mathrm{K}(x)} \phi_{i}\left(\delta_{x}\right)=\sum_{i \in N \backslash \Sigma(x)} \phi_{i}\left(\delta_{x}\right)
$$

The absolute efficiency axiom says that the total importance of attributes in the kernel equals the total importance of attributes outside the support. This property comes from the following fact. Considering $x \in L$ as above, by (17), the signed importance index yields

$$
0=\sum_{i \in N} \phi_{i}^{\mathrm{s}}\left(\delta_{x}\right)=\sum_{i \in \mathrm{K}(x)} \phi_{i}^{\mathrm{s}}\left(\delta_{x}\right)+\sum_{i \in N \backslash \Sigma(x)} \phi_{i}^{\mathrm{s}}\left(\delta_{x}\right)
$$

because for any $i \in \Sigma(x) \backslash \mathrm{K}(x), \phi_{i}^{\mathrm{s}}\left(\delta_{x}\right)=0$. This implies $\sum_{i \in \mathrm{K}(x)} \phi_{i}^{\mathrm{s}}\left(\delta_{x}\right)=-\sum_{i \in N \backslash \Sigma(x)} \phi_{i}^{\mathrm{s}}\left(\delta_{x}\right)$. Now, for the absolute importance index, we should consider absolute differences instead of signed ones, which immediately gives (AE), because $\phi_{i}^{\mathrm{s}}\left(\delta_{x}\right)>0$ for $i \in \mathrm{K}(x)$ and $\phi_{i}^{\mathrm{s}}\left(\delta_{x}\right)<0$ for $i \in N \backslash \Sigma(x)$.

Calibration axiom (C): For every $x \in L$ such that $N \backslash \Sigma(x)=\varnothing$,

$$
\sum_{i \in K(x)} \phi_{i}\left(\delta_{x}\right)=1
$$

The calibration axiom addresses the case (uncovered by the two previous axioms) where there is no attribute outside the support of the Dirac game. Then again, all attributes in the kernel have equal importance, whose sum is normalized to 1.

Theorem 3. Under axioms (CC), (I), (SD), (AE), (MC) and (C), for all $v \in \mathcal{G}(L)$ and all $i \in N$,

$$
\phi_{i}(v)=\phi_{i}^{\mathrm{a}}(v):=\sum_{\substack{x \in L \\ x_{i}<k_{i}}} \frac{\left(n-\sigma\left(x_{-i}\right)-1\right) ! \kappa\left(x_{-i}\right) !}{\left(n+\kappa\left(x_{-i}\right)-\sigma\left(x_{-i}\right)\right) !}\left|v\left(x+1_{i}\right)-v(x)\right| .
$$

The logical independence of axioms is shown in Appendix C. 


\section{Norm-based importance indices}

We now turn to the family of norm-based importance indices, which have the general form (10). As explained in Section 3, they appear as a natural generalization of absolute importance indices, which are based on the $L_{1}$ norm or its weighted version.

A fundamental difficulty is that the use of a norm different from $L_{1}$ forbids to take an axiomatic approach similar to the one we used in Section 6 , because there would exist no class of games where a property similar to linearity would hold. Nevertheless, it is possible to obtain a general form through a number of axioms which are presented below. In the rest of this section $i \in N$ is fixed.

Nonnegativity (NN): The importance index takes nonnegative values, i.e., $\phi_{i}$ : $\mathcal{G}(L) \rightarrow \mathbb{R}_{+}$.

Absolute Homogeneity (AH): For every $\alpha \in \mathbb{R}$ and every game $v \in \mathcal{G}(L)$,

$$
\phi_{i}(\alpha v)=|\alpha| \phi_{i}(v)
$$

Subadditivity (SA): For any games $v, w \in \mathcal{G}(L)$,

$$
\phi(v+w) \leq \phi(v)+\phi(w)
$$

Strong Null axiom (SN): $\phi_{i}(v)=0$ if and only if $i$ is null for $v$.

These axioms come directly from the definition of a norm, however, they are easily interpretable in our MCDA context. The nonnegativity axiom says that importance indices are nonnegative quantities. Absolute homogeneity says that multiplying a game by a constant just multiplies the importance index by the magnitude of this constant (as the importance index should be nonnegative). The subadditivity axiom expresses the fact that summing two games $v, w$ may mask the importance of an attribute: the positive variation of $i$ at some point $x$ for $v$ can be cancelled by a negative variation at the same point for $w$. Hence the overall variation for $v+w$ can be smaller than the sum of variations for $v, w$. Lastly, the strong null axiom is a strong version of the usual null axiom, in the sense that only games whose attribute $i$ is null lead to a null importance index for $i$.

We obtain the following.

Theorem 4. Under axioms (NN), (AH), (SA) and (SN), there exists a norm $\|\cdot\|$ on $\mathbb{R}^{k(k+1)^{n-1}}$ and a linear one-to-one mapping $h$ on $\mathbb{R}^{k(k+1)^{n-1}}$ such that

$$
\phi_{i}(v)=\left\|h \circ \Delta_{i}(v)\right\| .
$$

Additional axioms may be used to determine a particular norm or class of norms. Note however that the precise determination of $h$ through calibration or efficiency axioms seems to be difficult as $h$ lies inside the norm.

Note that since the four axioms (NN), (AH), (SA) and (SN) come directly from the definition of a norm, they must be then independent.

\section{Discussion and examples}

We apply Theorem 1 and Theorem 3 with the following examples. 
Example 2. We give a practical example of computation of $\phi_{i}^{\mathrm{s}}$ and $\phi_{i}^{\mathrm{a}}$ to the Dirac and unanimity games. Let $x \in L \backslash\left\{0_{N}\right\}$ and $i \in N$.

- The computation of $\phi_{i}$ w.r.t. $\delta_{x}$ gives

$$
\begin{gathered}
\phi_{i}^{\mathrm{S}}\left(\delta_{x_{-i}, 0}\right)=-\phi_{i}^{\mathrm{S}}\left(\delta_{x_{-i}, k_{i}}\right)=-\frac{\left(n-\sigma\left(x_{-i}\right)-1\right) ! \kappa\left(x_{-i}\right) !}{\left(n+\kappa\left(x_{-i}\right)-\sigma\left(x_{-i}\right)\right) !}, \text { and } \phi_{i}\left(\delta_{x_{-i}, x_{i}}\right)=0, \forall x_{i} \notin\left\{0, k_{i}\right\} . \\
\phi_{i}^{\mathrm{a}}\left(\delta_{x_{-i}, 0}\right)=\phi_{i}^{\mathrm{a}}\left(\delta_{x_{-i}, k_{i}}\right)=\phi_{i}^{\mathrm{s}}\left(\delta_{x_{-i}, k_{i}}\right), \text { and } \phi_{i}^{\mathrm{a}}\left(\delta_{x_{-i}, x_{i}}\right)=2 \phi_{i}^{\mathrm{a}}\left(\delta_{x_{-i}, k_{i}}\right), \forall x_{i} \notin\left\{0, k_{i}\right\} .
\end{gathered}
$$

- The computation of $\phi_{i}$ w.r.t. $u_{x}$ gives,

$$
\phi_{i}^{\mathrm{s}}\left(u_{x}\right)=\phi_{i}^{\mathrm{a}}\left(u_{x}\right)=\sum_{\substack{y_{-i} \in L_{-i} \\ y_{-i} \geq x_{-i}}} \frac{\left(n-\sigma\left(y_{-i}\right)-1\right) ! \kappa\left(y_{-i}\right) !}{\left(n+\kappa\left(y_{-i}\right)-\sigma\left(y_{-i}\right)\right) !}, \forall x_{i} \neq 0 .
$$

Let us take for example $N=\{1,2,3\}, k_{N}=(2,2,3)$, and $x=(0,1,3)$, we have,

$$
\begin{aligned}
\phi_{1}^{\mathrm{s}}\left(\delta_{x}\right)=-\frac{1}{2}, & \phi_{2}^{\mathrm{s}}\left(\delta_{x}\right)=0, & \phi_{3}^{\mathrm{s}}\left(\delta_{x}\right)=\frac{1}{2}, \\
\phi_{1}^{\mathrm{a}}\left(\delta_{x}\right)=\frac{1}{2}, & \phi_{2}^{\mathrm{a}}\left(\delta_{x}\right)=1, & \phi_{3}^{\mathrm{a}}\left(\delta_{x}\right)=\frac{1}{2}, \\
\phi_{1}^{\mathrm{s}}\left(u_{x}\right)=0, & \phi_{2}^{\mathrm{s}}\left(u_{x}\right)=1, & \phi_{3}^{\mathrm{s}}\left(u_{x}\right)=3 .
\end{aligned}
$$

We note that $\phi_{i}^{\mathrm{s}}\left(\delta_{x}\right)$ can be null when the peak is attained inside the domain $(i=2)$, is strictly negative if the peak is attained at $0(i=1)$, and is strictly positive if the peak is attained at the maximal value $k_{i}(i=3)$. However, $\phi_{i}^{\mathrm{a}}\left(\delta_{x}\right)$ is always strictly positive.

These results are in accordance with the theory, i.e., they illustrate in particular the respective efficiency and calibration axioms $(\mathbf{E}),(\mathbf{A E}),(\mathbf{C})$. Also, they are intuitively appealing:

(i) The absolute importance index accounts for the cumulated absolute variations along some axis. For the game $\delta_{x}$, its value changes along attributes 1 and 3 only once, while it changes twice for attribute 2 . This is exactly reflected by $\phi^{\mathrm{a}}$.

(ii) The signed importance index accounts for the average monotonicity of the function along some axis. For attribute $1, \delta_{x}(z)$ is decreasing along $z_{1}$, hence $\phi_{1}^{\mathrm{s}}\left(\delta_{x}\right)<0$, while it is increasing along attribute 3 . Now, when $z_{2}$ goes from 0 to $2, \delta_{x}(z)$ is first increasing then decreasing of the same amount. Thus, the overall variation is 0 . As a conclusion, the signed importance index should be more interpreted as an indicator of overall tendency of monotonicity, rather than an importance index.

(iii) Unanimity games being monotone, the two importance indices coincide. Attribute 1 has a null importance index because $u_{x}$ is constant w.r.t. attribute 1 . Attribute 3 has a greater importance index than attribute 2 because there is a variation of $u_{x}(z)$ along the second attribute $z_{2}$ only if $z_{3}=3$, while there is variation along $z_{3}$ for $z_{2}=1,2$. 
Example 3 (Thermal comfort). Thermal comfort is a subjective judgment depending on humidity $\left(X_{1}\right)$, temperature $\left(X_{2}\right)$ and air velocity $\left(X_{3}\right)$ among other parameters. The concept of thermal comfort has been studied from a "thermal" exchange standpoint (Fanger, 1970; Haldi and Robinson, 2011). The Predicted Mean Vote (PMV) is an assessment of the mean thermal feeling of individuals, on a bipolar scale taking 5 levels (from "very cold" to "very hot"). It is based on four thermal ambient condition factors, namely, the ambient air temperature $T a$ in ${ }^{\circ} \mathrm{C}$, the ambient air velocity $\mathrm{Va}$ in $\mathrm{m} / \mathrm{s}$, the rate of moisture in the air (humidity) $H y$ in \%, and the radiant temperature $\operatorname{Tr}$ in ${ }^{\circ} \mathrm{C}$, and on two personal parameters related to each person, namely, the metabolic coefficient $\mathrm{Me}$, and the clothing insulation $\mathrm{Ci}$ (Haldi and Robinson, 2011; Denguir, 2014). For the purpose of this paper, we can focus on the three most influential variables $\mathrm{Ta}, \mathrm{Hy}$ and $\mathrm{Va}$. The Predicted Percentage Dissatisfied (PPD) is a transformation of the PMV into a $[0,100]$ scale where 0 means that there is no dissatisfaction and 100 means that there is a complete dissatisfaction (Fanger, 1970). Figure 1 (resp. 2) shows PPD as a function of $\mathrm{Ta}$ and $\mathrm{Hy}$ (resp. $\mathrm{Ta}$ and $\mathrm{Va}$ ).

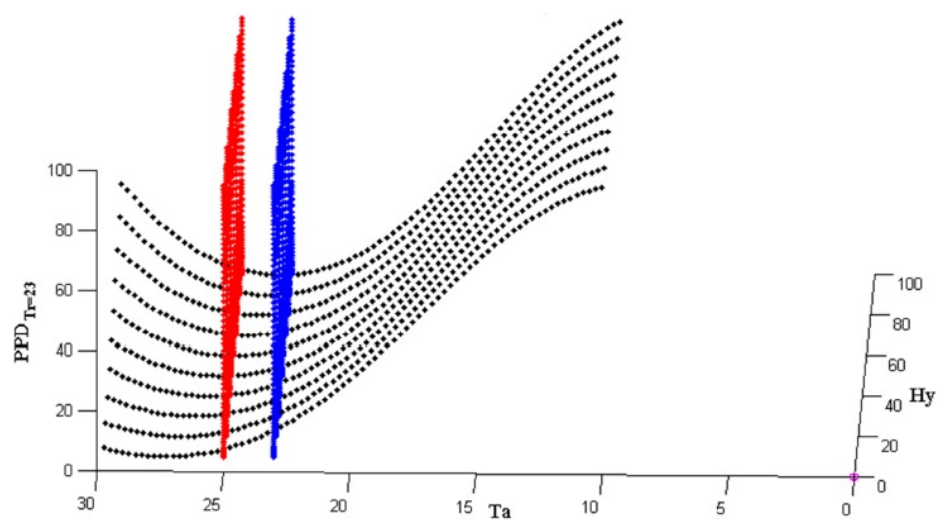

Figure 1: This picture represents the value of PPD as a function of $T a$, for different values of $H y$. Parameter $V a$ is fixed to 0.2. The ten curves correspond to 10 fixed values of $H y$ from $0 \%$ to $100 \%$. The red plane (resp. blue) cuts the PPD curves at value $T a=25^{\circ} \mathrm{C}$ (resp. $T a=23^{\circ} \mathrm{C}$ ). These planes clearly show that the value of $\mathrm{Ta}$ minimizing PPD depends on $\mathrm{Hy}$. Source (Denguir, 2014, Fig 10).

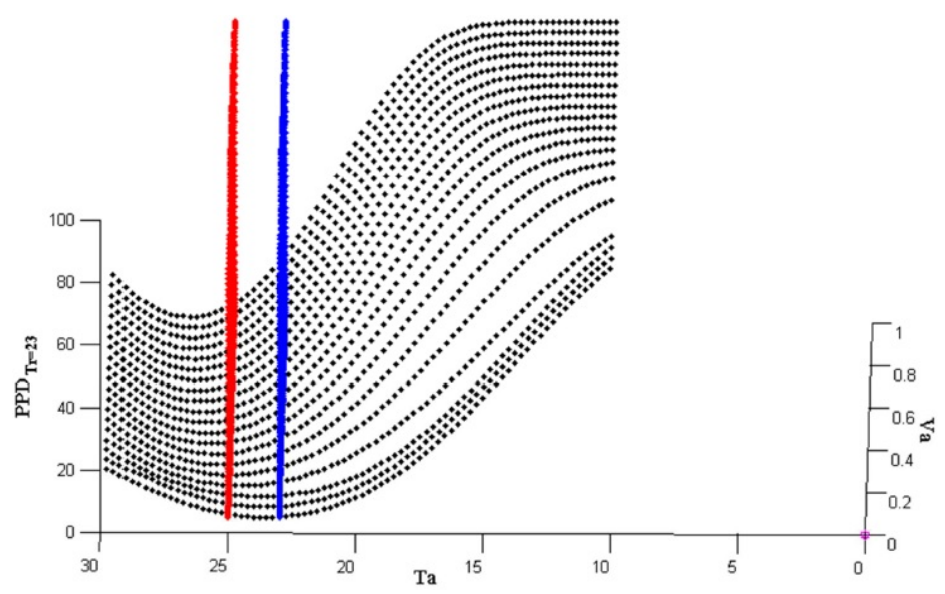

Figure 2: This picture presents PPD, as in Fig 1. More specifically, $H y$ is now fixed to $50 \%$, the ten curves represent PPD as a function of $T a$, for ten values of $V a$ from 0 to 1 . Source (Denguir, 2014, Fig 11). 
We consider that the function $U$ measures the discomfort level over the set $X=X_{1} \times X_{2} \times X_{3}$ defined by (Figure 3):

\begin{tabular}{|l|l|l|}
\hline$U(0,23,0)=2$ & $U(0,25,0)=4$ & $U(0,28,0)=7$ \\
$U(50,23,0)=5$ & $U(50,25,0)=7$ & $U(50,28,0)=10$ \\
$U(100,23,0)=10$ & $U(100,25,0)=12$ & $U(100,28,0)=15$ \\
\hline$U(0,23,0.2)=10$ & $U(0,25,0.2)=7$ & $U(0,28,0.2)=15$ \\
$U(50,23,0.2)=30$ & $U(50,25,0.2)=35$ & $U(50,28,0.2)=45$ \\
$U(100,23,0.2)=65$ & $U(100,25,0.2)=70$ & $U(100,28,0.2)=85$ \\
\hline$U(0,23,1)=40$ & $U(0,25,1)=35$ & $U(0,28,1)=30$ \\
$U(50,23,1)=87$ & $U(50,25,1)=75$ & $U(50,28,1)=70$ \\
$U(100,23,1)=97$ & $U(100,25,1)=90$ & $U(100,28,1)=83$ \\
\hline
\end{tabular}

We define the multichoice game $v \in \mathcal{G}(L)$ with $L=\{0,1,2\}^{3}$ by

$$
v(x)=U(Z(x))-U(Z(0,0,0)) \text {, for any } x \in L,
$$

where $Z$ is a transformation of the elements of $L$ in $X$ :

\begin{tabular}{|l|l|l|}
\hline$Z(0,0,0)=(0,23,0)$ & $Z(0,1,0)=(0,25,0)$ & $Z(0,2,0)=(0,28,0)$ \\
$Z(1,0,0)=(50,23,0)$ & $Z(1,1,0)=(50,25,0)$ & $Z(1,2,0)=(50,28,0)$ \\
$Z(2,0,0)=(100,23,0)$ & $Z(2,1,0)=(100,25,0)$ & $Z(2,2,0)=(100,28,0)$ \\
\hline$Z(0,0,1)=(0,23,0.2)$ & $Z(0,1,1)=(0,25,0.2)$ & $Z(0,2,1)=(0,28,0.2)$ \\
$Z(1,0,1)=(50,23,0.2)$ & $Z(1,1,1)=(50,25,0.2)$ & $Z(1,2,1)=(50,28,0.2)$ \\
$Z(2,0,1)=(100,23,0.2)$ & $Z(2,1,1)=(100,25,0.2)$ & $Z(2,2,1)=(100,28,0.2)$ \\
\hline$Z(0,0,2)=(0,23,1)$ & $Z(0,1,2)=(0,25,1)$ & $Z(0,2,2)=(0,28,1)$ \\
$Z(1,0,2)=(50,23,1)$ & $Z(1,1,2)=(50,25,1)$ & $Z(1,2,2)=(50,28,1)$ \\
$Z(2,0,2)=(100,23,1)$ & $Z(2,1,2)=(100,25,1)$ & $Z(2,2,2)=(100,28,1)$ \\
\hline
\end{tabular}

It is clear that the function $v$ is not monotone in its three arguments.

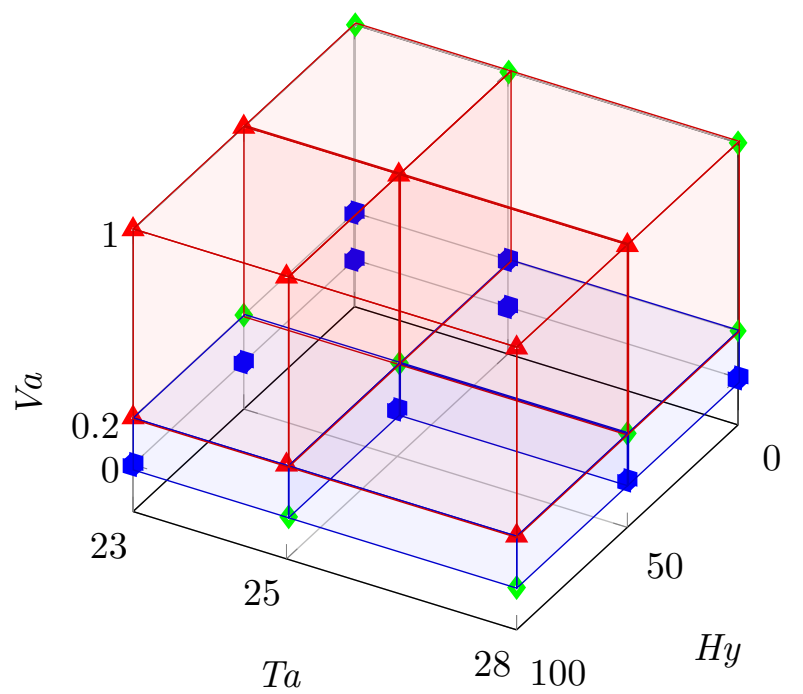

Figure 3: Discomfort level w.r.t. $X_{1}, X_{2}$ and $X_{3}$. Blue cube $=$ comfortable $([0,10])$, green diamond=fairly comfortable $([10,60])$, red triangle $=$ uncomfortable (] $60,100])$. 
The computation of the importance index w.r.t. $v$ gives,

$$
\begin{aligned}
& \phi_{1}^{\mathrm{s}}(v) \approx 188.17, \quad \phi_{2}^{\mathrm{s}}(v) \approx 17.67, \quad \phi_{3}^{\mathrm{s}}(v) \approx 247.17, \\
& \phi_{1}^{\mathrm{a}}(v) \approx 188.17, \quad \phi_{2}^{\mathrm{a}}(v) \approx 50.33, \quad \phi_{3}^{\mathrm{a}}(v) \approx 248.50 .
\end{aligned}
$$

The values of the absolute importance index show that air velocity is the most important criterion, then humidity, which both seem to be fairly more important than temperature. This slightly surprising conclusion may be explained by the fact that humidity variations (between 0 and 1) induce large variations in $U$ (e.g., $U(0,23,0)=2=U(0,23,1) / 20$,$) while temperature$ variations (between 23 and 28) induce smaller variations in $U$ (e.g., $U(0,23,0)=U(0,28,0) / 3.5$ ). Hence, the conclusion should be rather that, within the interval [23,28], temperature is relatively less important than humidity and air velocity.

The value of the signed importance index coincides with the absolute importance index for humidity since in the interval of temperature considered, the discomfort is increasing w.r.t. this attribute. The conclusion is almost the same for air velocity: the discomfort is increasing on most of the domain, except for high values, where it slightly decreases. By contrast, it can be checked on the table that the temperature has a more complex behavior. Depending on the values of the two other attributes, the discomfort is sometimes increasing, sometimes decreasing, and sometimes has a bell shape w.r.t. temperature. This is why the signed and the absolute importance indices differ, and the former one indicates that there is a slight overall tendency for the discomfort to augment with temperature.

\section{The continuous case}

In the continuous case of an aggregation function $F$, we have seen that a natural definition of the importance index is given by (7). It has been shown that this formula applied on $[0,1]^{n}$ yields the Shapley value when the aggregation function is the Choquet integral (see Marichal (1998, proposition 5.3.3 page 141) and also Grabisch et al. (2009, Definition 10.41 and Proposition 10.43 page 369$)$ ). We generalize this result in this section.

\subsection{Definition of the Choquet integral}

We consider first a normalized (1-ary) capacity on $N$, that is, a set function $\mu: 2^{N} \rightarrow \mathbb{R}$ satisfying monotonicity $(\mu(A) \leq \mu(B)$ whenever $A \subseteq B)$, and the normalization conditions $\mu(\emptyset)=0, \mu(N)=1$. The set of permutations on $N$ is denoted by $\mathfrak{S}$. For $\sigma \in \mathfrak{S}$, we define $\Omega_{\sigma}=\left\{t \in \mathbb{R}^{N}: t_{\sigma(1)} \leq t_{\sigma(2)} \leq \cdots \leq t_{\sigma(n)}\right\}$. The Choquet integral of $t \in[0,1]^{N}$ can be written in terms of the capacity $\mu$ (Choquet, 1953)

$$
C_{\mu}(t)=\sum_{i=1}^{n}\left(t_{\sigma(i)}-t_{\sigma(i-1)}\right) \mu(\{\sigma(i), \cdots, \sigma(n)\}),
$$

where $t_{\sigma(0)}:=0$ and $\sigma$ is a permutation on $N$ such that $t \in \Omega_{\sigma}$.

The Choquet integral can also be defined w.r.t. a $k_{N}$-ary capacity $v: L \rightarrow \mathbb{R}$. For $x \in$ $X:=\times_{i \in N}\left[0, k_{i}\right]$, we define $q_{i}(x) \in L_{i}$ by $q_{i}(x) \leq x_{i}<q_{i}(x)+1$ if $x_{i}<k_{i}$, and $q_{i}\left(k_{i}\right)=k_{i}-1$. We set $q(x)=\left(q_{1}(x), \ldots, q_{n}(x)\right)$. Point $x$ lies in the $n$-dimensional interval $\left[q(x), q(x)+1_{N}\right]$. The Choquet integral at $x$ w.r.t. $v$ is then simply the usual Choquet integral (24) at point $x-q(x) \in[0,1]^{n}$ w.r.t. a game $\mu_{q(x)}^{v}$, defined by

$$
\mu_{q}^{v}(S)=v\left((q+1)_{S}, q_{N \backslash S}\right)-v(q) .
$$


Then the Choquet integral of $x$ w.r.t. $v$ is defined by (Grabisch and Labreuche, 2003, 2008; Labreuche and Grabisch, 2018)

$$
\mathcal{C}_{v}(x)=C_{\mu_{q(x)}^{v}}(x-q(x))+v(q(x)) .
$$

\subsection{Computation of the importance indices}

For an aggregation function $F$ defined on an interval $\left[0, k_{N}\right]=\times_{i \in N}\left[0, k_{i}\right]$, we can define the signed and absolute importance of criterion $i$ by

$$
\begin{aligned}
\text { Signed importance: } \psi_{i}^{\mathrm{s}}(F) & =\int_{\left[0, k_{N}\right]} \frac{\partial F(x)}{\partial x_{i}} d x, \\
\text { Absolute importance: } \psi_{i}^{\mathrm{a}}(F) & =\int_{\left[0, k_{N}\right]}\left|\frac{\partial F(x)}{\partial x_{i}}\right| d x,
\end{aligned}
$$

The next result relates the importance indices on $v$ with the indices defined on aggregation functions.

Theorem 5. For every $i \in N$,

$$
\begin{aligned}
& \psi_{i}^{\mathrm{s}}\left(\mathcal{C}_{v}\right)=\phi_{i}^{\mathrm{s}}(v), \text { for any } k_{N} \text {-ary capacity } v \\
& \psi_{i}^{\mathrm{a}}\left(\mathcal{C}_{v}\right)=\phi_{i}^{\mathrm{a}}(v), \text { for any } v \in \mathcal{G}(L) .
\end{aligned}
$$

\section{Another axiomatization}

Lemma 3 in the proof of Theorem 1 shows that the signed importance index can be written as a sum over the grid of the Shapley value of local classical games limited to one elementary cell of the grid. The next axiomatization follows this scheme by considering that the domain can be decomposed into cells (axiom (DD)) and that the computation in one cell is invariant by translation (axiom $(\mathbf{T}))$.

For any $a=\left(a_{1}, \ldots, a_{n}\right)$ and $b=\left(b_{1}, \ldots, b_{n}\right) \in \mathbb{N}_{0}^{n}$, with $\mathbb{N}_{0}=\mathbb{N} \cup\{0\}$, such that $a_{i}<b_{i}$ for all $i \in N$, define $L_{i}^{a_{i}, b_{i}}=\left\{a_{i}, a_{i}+1, \ldots, b_{i}\right\}$ and $L^{a, b}=\times_{i \in N} L_{i}^{a_{i}, b_{i}}$.

In this section, we consider a multichoice game as a mapping $v$ on $L^{m, M}$ such that $v(m)=0$, with $m=\left(m_{1}, \ldots, m_{n}\right)$ and $M=\left(M_{1}, \ldots, M_{n}\right) \in \mathbb{N}_{0}^{n}$ indicating the lower bound and upper bound on each attribute. We denote by $\mathcal{G}\left(L^{m, M}\right)$ the set of multichoice games on $L^{m, M}$. For $v \in \mathcal{G}\left(L^{m, M}\right)$ and any $a, b \in \mathbb{N}_{0}^{n}$ such that $m_{i} \leq a_{i}<b_{i} \leq M_{i}$ for all $i$, we define the restriction $v^{\prime}:=v_{\mid L^{a, b}}$ in $\mathcal{G}\left(L^{a, b}\right)$ as $v^{\prime}(x)=v(x)$ for all $x \in L^{a, b}$.

The aim of this section is to define and axiomatize an importance index $\phi_{i}^{m, M}(v)$ for any attribute $i \in N$ and game $v \in \mathcal{G}\left(L^{m, M}\right)$. Note that $L=L^{0_{N}, k_{N}}$, and $\phi=\phi^{0_{N}, k_{N}}$, while letting $0_{N}=(0, \ldots, 0), 1_{N}=(1, \ldots, 1)$, a classical game is an element of $\mathcal{G}\left(L^{0_{N}, 1_{N}}\right)$.

The first axiom says that $\phi$ can be additively decomposed on the lattice $L^{m, M}$.

Decomposition over Domains (DD): Let $j \in N$ and $\ell \in \mathbb{N}$ such that $m_{j}<\ell<$ $M_{j}$.

Let $v \in \mathcal{G}\left(L^{m, M}\right)$. We define $v_{1}=v_{\mid L^{m,\left(\ell_{j}, M_{-j}\right)}}$ and $v_{2}=v_{\mid L^{\left(\ell_{j}, m_{-j}\right), M}}$. Then for every $i \in N$

$$
\phi_{i}^{m, M}(v)=\phi_{i}^{m,\left(\ell_{j}, M_{-j}\right)}\left(v_{1}\right)+\phi_{i}^{\left(\ell_{j}, m_{-j}\right), M}\left(v_{2}\right) .
$$


Remark 4. From DD applied $\prod_{i \in N}\left(M_{i}-m_{i}-1\right)$ times, we obtain

$$
\phi_{i}^{m, M}(v)=\sum_{y \in L^{m, M-1_{N}}} \phi_{i}^{y, y+1_{N}}\left(v_{\mid L^{y, y+1_{N}}}\right) .
$$

Linearity axiom $\left(\mathbf{L}_{01}\right): \phi^{0_{N}, 1_{N}}$ is linear on $\mathcal{G}\left(L^{0_{N}, 1_{N}}\right)$, i.e., $\forall v, w \in \mathcal{G}\left(L^{0_{N}, 1_{N}}\right), \forall \alpha \in$ $\mathbb{R}$

$$
\phi^{0_{N}, 1_{N}}(v+\alpha w)=\phi^{0_{N}, 1_{N}}(v)+\alpha \phi^{0_{N}, 1_{N}}(w) .
$$

Definition 1. An attribute $i \in N$ is said to be $n u l l$ for $v \in \mathcal{G}\left(L^{0_{N}, 1_{N}}\right)$ if

$$
v\left(x+1_{i}\right)=v(x) \text {, for every } x \in L^{0_{N}, 1_{N}} \text { such that } x_{i}=0 .
$$

Null axiom $\left(\mathbf{N}_{01}\right)$ : If an attribute $i$ is null for $v \in \mathcal{G}\left(L^{0_{N}, 1_{N}}\right)$, then

$$
\phi_{i}^{0_{N}, 1_{N}}(v)=0 .
$$

Equal Treatment $\left(\mathbf{E T}_{01}\right)$ : Let $v \in \mathcal{G}\left(L^{0_{N}, 1_{N}}\right)$ and $i, j \in N$.

If $v\left(x+1_{i}\right)=v\left(x+1_{j}\right)$ for all $x \in L^{0_{N}, 1_{N}}$ with $x_{i}=0$ and $x_{j}=0$, then

$$
\phi_{i}^{0_{N}, 1_{N}}(v)=\phi_{j}^{0_{N}, 1_{N}}(v) .
$$

$\operatorname{Efficiency}\left(\mathbf{E}_{01}\right): \forall v \in \mathcal{G}\left(L^{0_{N}, 1_{N}}\right), \sum_{i \in N} \phi_{i}^{0_{N}, 1_{N}}(v)=v(N)$.

The next axiom is a translation property. It says that the computation of the importance index in one elementary cell $L^{y, y+1_{N}}$ does not depend on the origin point $y$ : it can be obtained from the computation of the index in $L^{0_{N}, 1_{N}}$ cell by a shift operation on the game.

Translation axiom (T): For all $y \in L^{m, M}$, such that $y+1_{N} \leq M$, and for any $i \in N$,

$$
\phi_{i}^{y, y+1_{N}}(v)=\phi_{i}^{0_{N}, 1_{N}}\left(T_{y} \circ v\right),
$$

where $v \in \mathcal{G}\left(L^{y, y+1_{N}}\right)$ and $T_{y} \circ v$ is a game in $\mathcal{G}\left(L^{0_{N}, 1_{N}}\right)$ defined by $T_{y} \circ v(z)=$ $v(y+z)-v(y), \forall z \in L^{0_{N}, 1_{N}}$.

Theorem 6. Under axioms (DD), $\left(\mathbf{L}_{01}\right),\left(\mathbf{N}_{01}\right),(\mathbf{T}),\left(\mathbf{E T}_{01}\right)$ and $\left(\mathbf{E}_{01}\right), \forall v \in \mathcal{G}\left(L^{m, M}\right)$

$$
\phi_{i}^{m, M}(v)=\phi_{i}^{\mathrm{s} ; m, M}(v)=\sum_{x_{-i} \in L_{-i}^{m, M}} \frac{\left(n-\sigma\left(x_{-i}\right)-1\right) ! \kappa\left(x_{-i}\right) !}{\left(n+\kappa\left(x_{-i}\right)-\sigma\left(x_{-i}\right)\right) !}\left(v\left(x_{-i}, M_{i}\right)-v\left(x_{-i}, m_{i}\right)\right), \forall i \in N .
$$

We turn now to the axiomatization of the family defined by the absolute value.

Decomposition over Domains for Monotone games (DDM) $: \phi^{m, M}$ satisfies the (DD) axiom on $\mathcal{G}_{M}\left(L^{m, M}\right)$.

Conic Combination axiom $\left(\mathbf{C C}_{01}\right)$ : For every $v, w \in \mathcal{G}_{+}\left(L^{0_{N}}, 1_{N}\right)$, for every $\alpha \in \mathbb{R}_{+}$,

$$
\phi^{0_{N}, 1_{N}}(v+\alpha w)=\phi^{0_{N}, 1_{N}}(v)+\alpha \phi^{0_{N}, 1_{N}}(w) .
$$

Decomposition axiom $\left(\mathbf{D}_{01}\right)$ : If $v, w \in \mathcal{G}_{+}\left(L^{0_{N}, 1_{N}}\right)$ and $v-w \in \mathcal{G}_{M}\left(L^{0_{N}, 1_{N}}\right)$, then

$$
\phi^{0_{N}, 1_{N}}(v-w)=\phi^{0_{N}, 1_{N}}(v)-\phi^{0_{N}, 1_{N}}(w) .
$$


Efficiency for Monotone games $\left(\mathbf{E M}_{01}\right): \phi^{0_{N}, 1_{N}}$ satisfies the $\left(\mathrm{E}_{01}\right)$ axiom on $\mathcal{G}_{M}\left(L^{0_{N}, 1_{N}}\right)$.

We also use the marginal contribution axiom (MC) introduced in Section 6, but here for games in $\mathcal{G}\left(L^{m, M}\right)$.

Proposition 5. Under axioms (DDM), $\left(\mathbf{C C}_{01}\right),\left(\mathbf{D}_{01}\right),\left(\mathbf{N}_{01}\right),\left(\mathbf{E T}_{01}\right),(\mathbf{T}),\left(\mathbf{E M}_{01}\right)$ and (MC), $\forall v \in \mathcal{G}\left(L^{m, M}\right), \forall i \in N$,

$$
\phi_{i}^{m, M}(v)=\phi_{i}^{\mathrm{a} ; m, M}(v)=\sum_{\substack{x \in L^{m, M} \\ x_{i}<M_{i}}} \frac{\left(n-\sigma\left(x_{-i}\right)-1\right) ! \kappa\left(x_{-i}\right) !}{\left(n+\kappa\left(x_{-i}\right)-\sigma\left(x_{-i}\right)\right) !}\left|v\left(x+1_{i}\right)-v(x)\right| .
$$

\section{Concluding remarks}

We have presented in this paper two families of importance indices, the signed and the absolute importance indices, together with two remarkable representatives $\phi^{\mathrm{a}}, \phi^{\mathrm{s}}$. They are valid for any kind of numerical model of representation of preferences (monotonic or not, discrete or continuous), and we have characterized them through natural and interpretable axioms. Further generalization is possible through the use of norms, but still further investigation is necessary.

The proposed indices are close in spirit to the Shapley value, and a similar approach could also be undertaken using the Banzhaf value as starting point.

Both indices can be useful, and their combination may shed light on the model under analysis. The signed index indicates the overall trend of the model w.r.t. an attribute: increasing or decreasing. The absolute index measures the amount of variation of the model induced by an attribute. An attribute having a high value of the absolute importance index and a low value of the signed importance index indicates an attribute which has a strong contribution in the model, but the model is not monotonic w.r.t. this attribute: it could be bell-shaped or could have a more complex behavior. We have illustrated the usage of these indices on examples, among which a modelling of discomfort based on real data. Lastly, we mention that these indices contribute to the theory of multichoice games by providing a new type of Shapley value.

Our axiomatization results are summarized in the following table, in which a "+ " means that a value satisfies the axiom, while " - " has the converse meaning. Symbol " $\times$ " shows that the axiom is used for the axiomatization.

\begin{tabular}{|c|c|c|c|c|c|c|c|c|}
\hline & $\mathrm{L}$ & $\mathrm{I}$ & $\mathrm{SD}$ & $\mathrm{E}$ & $\mathrm{CC}$ & $\mathrm{MC}$ & $\mathrm{AE}$ & $\mathrm{C}$ \\
\hline$\phi^{\mathrm{s}}$ & $\times$ & $\times$ & $\times$ & $\times$ & + & - & - & + \\
\hline$\phi^{\mathrm{a}}$ & - & $\times$ & $\times$ & - & $\times$ & $\times$ & $\times$ & $\times$ \\
\hline
\end{tabular}

\section{Appendix A Proof of the results}

The following lemma is used to prove Proposition 1.

Lemma 1. Under axioms $(\mathbf{L})$ and $(\mathbf{N})$, for all $i \in N$, there exists $p_{x}^{i} \in \mathbb{R}$, for all $x \in L$ with $x_{i}<k_{i}$, such that $\forall v \in \mathcal{G}(L)$,

$$
\phi_{i}(v)=\sum_{\substack{x \in L \\ x_{i}<k_{i}}} p_{x}^{i}\left(v\left(x+1_{i}\right)-v(x)\right) .
$$


Proof. It is easy to check that the above formula satisfies the two axioms. Conversely, we consider $\phi$ satisfying (L) and (N). We have $v=\sum_{x \in L} v(x) \delta_{x}$. Then by (L),

$$
\phi_{i}(v)=\sum_{x \in L} v(x) \phi_{i}\left(\delta_{x}\right), \forall i \in N .
$$

Setting $a_{x}^{i}=\phi_{i}\left(\delta_{x}\right), \forall x \in L, \forall i \in N$, we obtain, assuming $i$ is null for $v$ :

$$
\begin{aligned}
0=\phi_{i}(v) & =\sum_{x \in L} a_{x}^{i} v(x) \\
& =\sum_{x_{-i} \in L_{-i}} \sum_{x_{i} \in L_{i}} a_{\left(x_{-i}, x_{i}\right)}^{i} v\left(x_{-i}, x_{i}\right) \\
& =\sum_{x_{-i} \in L_{-i}}\left(\sum_{x_{i} \in L_{i}} a_{\left(x_{-i}, x_{i}\right)}^{i}\right) v\left(x_{-i}, 0_{i}\right) .
\end{aligned}
$$

Hence, $\forall x_{-i} \in L_{-i}, \sum_{x_{i} \in L_{i}} a_{\left(x_{-i}, x_{i}\right)}=0$.

Letting $p_{\left(x_{-i}, j_{i}\right)}=\sum_{\ell=j+1}^{k} a_{\left(x_{-i}, \ell_{i}\right)}, \forall x_{-i} \in L_{-i}, \forall j \in L_{i} \backslash k_{i}$, we have, for all $x_{-i} \in L_{-i}$,

$$
\begin{aligned}
& a_{\left(x_{-i}, 0_{i}\right)}=-p_{\left(x_{-i}, 0_{i}\right)}, \\
& a_{\left(x_{-i}, k_{i}\right)}=p_{\left(x_{-i},\left(k_{i}-1\right)_{i}\right)}, \\
& a_{\left(x_{-i}, \ell_{i}\right)}=p_{\left(x_{-i},(\ell-1)_{i}\right)}-p_{\left(x_{-i}, \ell_{i}\right)}, \forall \ell_{i} \in L_{i} \backslash\left\{0, k_{i}\right\} .
\end{aligned}
$$

Therefore, it suffices to replace the values of $a_{\left(x_{-i}, x_{i}\right)}^{i}$ in Formula (34), and the result is established.

Proof of Proposition 1. It is easy to check that the given formula satisfies the axioms. Conversely, consider $\phi$ satisfying (L) and (I). Let $v, w \in \mathcal{G}(L)$ such that $v, w$ satisfy the premise of the Invariance axiom, and $i \in N$. By Lemma 1 and $(\mathbf{I})$, we have:

$$
\begin{aligned}
\phi_{i}(v) & =\sum_{\substack{x \in L \\
x_{i}<k_{i}}} p_{x_{i} ; x_{-i}}^{i}\left(v\left(x+1_{i}\right)-v(x)\right) \\
& =\sum_{x_{-i} \in L_{-i}}\left(\sum_{\substack{x_{i} \in L_{i} \\
x_{i} \notin\left\{0, k_{i}\right\}}} p_{x_{i} ; x_{-i}}^{i}\left(v\left(x+1_{i}\right)-v(x)\right)+p_{0_{i} ; x_{-i}}^{i}\left(v\left(x_{-i}, 1_{i}\right)-v\left(x_{-i}, 0_{i}\right)\right)\right) \\
& =\sum_{x_{-i} \in L_{-i}}\left(\sum_{\substack{x_{i} \in L_{i} \\
x_{i} \notin\left\{0, k_{i}\right\}}} p_{x_{i} ; x_{-i}}^{i}\left(w(x)-w\left(x-1_{i}\right)\right)+p_{0_{i} ; x_{-i}}^{i}\left(w\left(x_{-i}, k_{i}\right)-w\left(x_{-i},\left(k_{i}-1\right)_{i}\right)\right)\right) \\
& =\sum_{x_{-i} \in L_{-i}}\left(\sum_{\substack{x_{i} \in L_{i} \\
x_{i}<k_{i}-1}} p_{x_{i}+1 ; x_{-i}}^{i}\left(w\left(x+1_{i}\right)-w(x)\right)+p_{0_{i} ; x_{-i}}^{i}\left(w\left(x_{-i}, k_{i}\right)-w\left(x_{-i},\left(k_{i}-1\right)_{i}\right)\right)\right),
\end{aligned}
$$

and,

$\phi_{i}(w)=\sum_{x_{-i} \in L_{-i}}\left(\sum_{\substack{x_{i} \in L_{i} \\ x_{i}<k_{i}-1}} p_{x_{i} ; x_{-i}}^{i}\left(w\left(x+1_{i}\right)-w(x)\right)+p_{\left(k_{i}-1\right)_{i} ; x_{-i}}^{i}\left(w\left(x_{-i}, k_{i}\right)-w\left(x_{-i},\left(k_{i}-1\right)_{i}\right)\right)\right)$,

then, $p_{x_{i} ; x_{-i}}^{i}=p_{x_{i}+1 ; x_{-i}}^{i}, \forall x_{-i} \in L_{-i}, \forall x_{i} \in L_{i} \backslash\left\{k_{i}-1, k_{i}\right\}$.

We conclude that the coefficient $p_{x_{i} ; x_{-i}}^{i}$ does not depend on $x_{i}$. We denote by $p_{x_{-i}}^{i}$ this value. Therefore, it suffices to replace the values of $p_{x_{i} ; x_{-i}}^{i}$ in formula (33) by $p_{x_{-i}}^{i}$, and the result is established. 
The proof of Theorem 1 is based on the following lemmas:

\section{Lemma 2.}

$$
\sum_{S \in[A, B]} \frac{(n-s-1) ! s !}{n !}=\frac{(n-b-1) ! a !}{(n-b+a) !}, \forall A, B \subseteq N, A \subseteq B,
$$

where $[A, B]=\{C \subseteq N: A \subseteq C \subseteq B\}$.

Proof. Let $A, B \subseteq N$, such that $A \subseteq B$,

$$
\begin{aligned}
\sum_{S \in[A, B]} \frac{(n-s-1) ! s !}{n !} & =\sum_{S \in[\varnothing, B \backslash A]} \frac{(n-s-a-1) !(s+a) !}{n !} \\
& =\sum_{s=0}^{b-a}\left(\begin{array}{c}
b-a \\
s
\end{array}\right) \frac{(n-s-a-1) !(s+a) !}{n !} \\
& =\sum_{s=0}^{b-a}\left(\begin{array}{c}
b-a \\
s
\end{array}\right) \int_{0}^{1} x^{n-s-a-1}(1-x)^{s+a} d x \\
& =\int_{0}^{1} x^{n-b-1}(1-x)^{a} \sum_{s=0}^{b-a}\left(\begin{array}{c}
b-a \\
s
\end{array}\right) x^{b-a-s}(1-x)^{s} d x \\
& =\int_{0}^{1} x^{n-b-1}(1-x)^{a} d x \\
& =\frac{(n-b-1) ! a !}{(n-b+a) !}
\end{aligned}
$$

Lemma 3. Let $v \in \mathcal{G}(L)$.

$$
\phi_{i}^{\mathrm{s}}(v)=\sum_{\substack{x \in L \\ \forall j \in N, x_{j}<k_{j}}} \phi_{i}^{S h}\left(\mu_{x}^{v}\right), \forall i \in N,
$$

with, $\mu_{x}^{v}(S)=v\left(x+1_{S}\right)-v(x), \forall S \subseteq N, \forall x \in L$, such that $x_{i}<k_{i}, \forall i \in N$.

Proof. Let $v \in \mathcal{G}(L)$ and for any $x \in L$, such that $x_{i}<k_{i}, \forall i \in N$, we define the game $\mu_{x}^{v}$ such that for every $S \subseteq N, \mu_{x}^{v}(S)=v\left(x+1_{S}\right)-v(x)$. We have 


$$
\begin{aligned}
& \phi_{i}^{\mathrm{S}}(v)=\sum_{\substack{x \in L \\
x_{i}<k_{i}}} \frac{\left(n-\sigma\left(x_{-i}\right)-1\right) ! \kappa\left(x_{-i}\right) !}{\left(n+\kappa\left(x_{-i}\right)-\sigma\left(x_{-i}\right)\right) !}\left(v\left(x+1_{i}\right)-v(x)\right) \\
& =\sum_{\substack{x \in L \\
x_{i}<k_{i}}}\left(v\left(x+1_{i}\right)-v(x)\right) \sum_{\substack{A \subseteq \Sigma\left(x_{-i}\right) \\
A \supseteq \mathrm{K}\left(x_{-i}\right)}} \frac{(n-a-1) ! a !}{n !} \text { by Lemma (2) } \\
& =\sum_{\substack{x \in L \\
x_{i}<k_{i}}}\left(v\left(x+1_{i}\right)-v(x)\right) \sum_{\substack{A \subseteq N \backslash i \cap \Sigma\left(x_{-i}\right) \\
A \supseteq \mathrm{K}\left(x_{-i}\right)}} \frac{(n-a-1) ! a !}{n !} \\
& =\sum_{\substack{x \in L \\
x_{i}<k_{i}}}\left(v\left(x+1_{i}\right)-v(x)\right) \sum_{\substack{A \subseteq N \backslash i \\
\forall j \in A, x_{j}>0 \\
\forall j \in N \backslash A, x_{j}<k_{j}}} \frac{(n-a-1) ! a !}{n !} \\
& =\sum_{\substack{x \in L \\
\forall j \in N, x_{j}<k_{j}}} \sum_{S \subseteq N \backslash i} \frac{(n-s-1) ! s !}{n !}\left(v\left(x+1_{S \cup i}\right)-v\left(x+1_{S}\right)\right) \\
& \left.=\sum_{\substack{x \in L \\
\forall j \in N, x_{j}<k_{j}}} \sum_{S \subseteq N \backslash i} \frac{(n-s-1) ! s !}{n !}\left(\mu_{x}^{v}(S \cup i)-\mu_{x}^{v}(S)\right)\right) \\
& =\sum_{\substack{x \in L \\
\forall j \in N, x_{j}<k_{j}}} \phi_{i}^{S h}\left(\mu_{x}^{v}\right)
\end{aligned}
$$

We are now in position to show Theorem 1 .

Proof of Theorem 1. It is easy to check that the formula (19) satisfies the axioms (L), (I) and (SD). Let us show that it satisfies the axiom (E). By Lemma 3, we have,

$$
\begin{aligned}
\sum_{i \in N} \phi_{i}^{\mathrm{s}}(v)= & \sum_{\substack{x \in L \\
\forall j \in N, x_{j}<k_{j}}} \sum_{i \in N} \phi_{i}^{S h}\left(\mu_{x}^{v}\right) \\
= & \sum_{\substack{x \in L \\
\forall j \in N, x_{j}<k_{j}}} \mu_{x}^{v}(N) \\
= & \sum_{\substack{x \in L \\
\forall j \in N, x_{j}<k_{j}}}\left(v\left(x+1_{N}\right)-v(x)\right) .
\end{aligned}
$$

Conversely, we consider $\phi$ satisfying the axioms (L), (I), (SD) and (E). Let $x \in L$, we write $x=\left(0_{N \backslash S \cup T}, x_{S}, k_{T}\right)$, with $x_{S} \in L_{S} \backslash \Gamma\left(L_{S}\right), S=\Sigma(x) \backslash \mathrm{K}(x)$, and $T=\mathrm{K}(x)$.

By Proposition 1, we have,

$$
\phi_{i}\left(\delta_{x}\right)=p_{x_{-i}}^{i}\left(\delta_{x}\left(x_{-i}, k_{i}\right)-\delta_{x}\left(x_{-i}, 0_{i}\right)\right)
$$

from which we obtain

$$
\phi_{i}\left(\delta_{\left(x_{-i}, k_{i}\right)}\right)=p_{x_{-i}}^{i}=-\phi_{i}\left(\delta_{\left(x_{-i}, 0_{i}\right)}\right)
$$


and

$$
\phi_{i}\left(\delta_{\left(x_{-i}, x_{i}\right)}\right)=0 \text {, for } x_{i} \in L_{i} \backslash\left\{0, k_{i}\right\} .
$$

We only have to determine $\phi_{i}\left(\delta_{\left(x_{-i}, k_{i}\right)}\right)$, with $x_{-i}=\left(0_{N \backslash S \cup T}, x_{S}, k_{T \backslash i}\right)$.

We have

$$
\sum_{i \in N} \phi_{i}\left(\delta_{x}\right)=\sum_{i \in T} \phi_{i}\left(\delta_{x}\right)+\sum_{i \in S} \phi_{i}\left(\delta_{x}\right)+\sum_{i \in N \backslash S \cup T} \phi_{i}\left(\delta_{x}\right)
$$

and by (36), we obtain,

$$
\sum_{i \in N} \phi_{i}\left(\delta_{x}\right)=\sum_{i \in T} \phi_{i}\left(\delta_{x_{-i}, k_{i}}\right)+\sum_{i \in N \backslash S \cup T} \phi_{i}\left(\delta_{x_{-i}, 0_{i}}\right) .
$$

We distinguish the two following cases:

(i) If $S \cup T=N$,

$$
\sum_{j \in N} \phi_{j}\left(\delta_{x}\right)=(n-s) \phi_{i}\left(\delta_{x_{-i}, k_{i}}\right), \forall i \in T
$$

(ii) If $S \cup T \neq N$,

$$
\sum_{\ell \in N} \phi_{\ell}\left(\delta_{x}\right)=t \phi_{i}\left(\delta_{x_{-i}, k_{i}}\right)+(n-s-t) \phi_{j}\left(\delta_{x_{-j}, 0_{j}}\right), \forall i \in T, \forall j \in N \backslash S \cup T .
$$

Hence, $\phi_{i}\left(\delta_{\left(x_{-i}, k_{i}\right)}\right)$ satisfies

- by (38) and (15),

$$
\forall i \in \mathrm{K}(x),(\text { with } \sigma(x)=n), \phi_{i}\left(\delta_{\left(x_{-i}, k_{i}\right)}\right)=\frac{1}{n-s}, \forall s \in\{0, \ldots, n-1\},
$$

- by (39) and (17), $\forall t \in\{1, \ldots, n-1\}, \forall s \in\{0, \ldots, n-1\}$ with $s+t \leq n-1$,

$$
\phi_{i}\left(\delta_{\left(x_{-i j}, 0_{j}, k_{i}\right)}\right)=-\frac{n-s-t}{t} \phi_{j}\left(\delta_{\left(x_{-i j}, 0_{j}, k_{i}\right)}\right),
$$

and by (35), we obtain

$$
\phi_{i}\left(\delta_{\left(x_{-i j}, 0_{j}, k_{i}\right)}\right)=\frac{n-s-t}{t} \phi_{j}\left(\delta_{\left(x_{-i j}, k_{j}, k_{i}\right)}\right) .
$$

Thus, recursively,

$$
\begin{aligned}
\phi_{i}\left(\delta_{\left(0_{N \backslash S \cup T}, x_{S}, k_{T \backslash i}, k_{i}\right)}\right) & =\frac{n-s-t}{t} \phi_{j}\left(\delta_{\left(0_{N \backslash S \cup T_{1}}, x_{S}, k_{T_{1}}\right)}\right), T_{1}=T \cup\{j\} \\
\phi_{i}\left(\delta_{\left(0_{N \backslash S \cup T_{1}}, x_{S}, k_{T_{1} \backslash i}, k_{i}\right)}\right) & =\frac{n-s-t-1}{t+1} \phi_{j}\left(\delta_{\left(0_{N \backslash S \cup T_{2}}, x_{S}, k_{T_{2}}\right)}\right), T_{2}=T_{1} \cup\{j\} \\
& \vdots \\
\phi_{i}\left(\delta_{\left(0_{j}, x_{S}, k_{T_{n-s-1} \backslash i}, k_{i}\right)}\right) & =\frac{1}{n-s-1} \phi_{j}\left(\delta_{\left(x_{S}, k_{T_{n-s}}\right)}\right), T_{n-s}=T_{n-s-1} \cup\{j\} .
\end{aligned}
$$


Therefore,

$$
\begin{aligned}
\phi_{i}\left(\delta_{\left(x_{-i}, k_{i}\right)}\right) & =\frac{(n-s-t) !(t-1) !}{(n-s) !} \\
& =\frac{(n-\sigma(x)) !(\kappa(x)-1) !}{(n+\kappa(x)-\sigma(x)) !} \\
& =\frac{\left(n-\sigma\left(x_{-i}, k_{i}\right)\right) !\left(\kappa\left(x_{-i}, k_{i}\right)-1\right) !}{\left(n+\kappa\left(x_{-i}, k_{i}\right)-\sigma\left(x_{-i}, k_{i}\right)\right) !} \\
& =\frac{\left(n-\sigma\left(x_{-i}\right)-1\right) !\left(\kappa\left(x_{-i}\right) !\right.}{\left(n+\kappa\left(x_{-i}\right)-\sigma\left(x_{-i}\right)\right) !}
\end{aligned}
$$

The result is proved.

Proof of Proposition 2. By using the basis of unanimity games, and by the definition of $\mathcal{G}_{+}(L)$,

$$
v=\sum_{x \in L} m^{v}(x) u_{x}
$$

for any $v \in \mathcal{G}_{+}(L)$, and $m^{v}(x) \geq 0$. Applying (CC) we find

$$
\phi_{i}(v)=\sum_{x \in L} m^{v}(x) \phi_{i}\left(u_{x}\right) \quad(i \in N) .
$$

Since $m^{v}$ is a linear transform over $\mathcal{G}(L), m^{v}(x)$ is a linear combination of all coefficients $v(y)$, $y \in L$, say $m^{v}(x)=\sum_{y \in L} a(x, y) v(y)$. Then

$$
\begin{aligned}
\phi_{i}(v) & =\sum_{x \in L}\left(\sum_{y \in L} a(x, y) v(y)\right) \phi_{i}\left(u_{x}\right) \\
& =\sum_{y \in L} v(y) \underbrace{\sum_{x \in L} a(x, y) \phi_{i}\left(u_{x}\right)}_{a_{y}^{i}}
\end{aligned}
$$

giving the desired form, where $a_{y}^{i}$ are real constants independent from $v$. Consider now a game $v \in \mathcal{G}_{M}(L)$. Applying (6), we write $v=v^{+}-v^{-}$, and by (CC) we find

$$
\phi_{i}(v)=\phi_{i}\left(v^{+}-v^{-}\right)=\phi_{i}\left(v^{+}\right)-\phi_{i}\left(v^{-}\right)=\sum_{x \in L} a_{x}^{i} v^{+}(x)-\sum_{x \in L} a_{x}^{i} v^{-}(x)=\sum_{x \in L} a_{x}^{i} v(x) .
$$

The proof of Proposition 3 is based on the following lemma

Lemma 4. Let $i \in N$. For every $v \in \mathcal{G}(L)$, there exists $w \in \mathcal{G}_{M}(L)$, such that $\left|\Delta_{i} v\right|=\left|\Delta_{i} w\right|$.

Proof. Let $v \in \mathcal{G}(L)$ and $i \in N$. For every $y_{-i} \in L_{-i}$ we set,

$$
D\left(x_{i}, y_{-i}\right)=\sum_{\substack{z_{i} \in L_{i} \\ z_{i}<x_{i}}}\left|\Delta_{i} v\left(z_{i}, y_{-i}\right)\right|, \forall x_{i} \in L_{i} \backslash\{0\}, \text { and } D\left(0_{i}, y_{-i}\right)=0 \text {. }
$$


Define the following multichoice game $w$ :

$$
\begin{aligned}
w(0, \ldots, 0) & =0 \\
w\left(0_{i}, x_{-i}\right) & =\max _{\substack{x_{i} \in L_{i} \\
y_{-i} \in L_{-i}, y_{-i} \leq x_{-i}}}\left(w\left(0_{i}, y_{-i}\right)+D\left(x_{i}, y_{-i}\right)-D\left(x_{i}, x_{-i}\right)\right), \quad x_{-i} \in L_{-i} \\
w\left(x_{i}, y_{-i}\right) & =w\left(0_{i}, y_{-i}\right)+D\left(x_{i}, y_{-i}\right), \quad x_{i}>0, y_{-i} \in L_{-i} .
\end{aligned}
$$

Then by construction we have $w\left(x+1_{i}\right)=w(x)+\left|\Delta_{i} v(x)\right|, \forall x \in L, x_{i}<k_{i}$. We prove now monotonocity. Let $j \in N \backslash i$, and $x \in L$ such that $x_{j}<k_{j}$. If $x_{i}=0$, we have for every $y \in L$ such that $y_{-i} \lesseqgtr x_{-i}+1_{j}$,

$$
w\left(0_{i}, x_{-i}+1_{j}\right) \geq w\left(0_{i}, y_{-i}\right)+D(y)-D\left(y_{i}, x_{-i}+1_{j}\right) .
$$

Taking $y_{i}=0$ and $y_{-i}=x_{-i}$, we have

$$
w\left(0_{i}, x_{-i}+1_{j}\right) \geq w\left(0_{i}, x_{-i}\right) .
$$

If now $x_{i} \neq 0$, we have for every $y \in L$ such that $y_{-i} \lesseqgtr x_{-i}+1_{j}$,

$$
w\left(x+1_{j}\right) \geq w\left(0_{i}, y_{-i}\right)+D(y)-D\left(y_{i}, x_{-i}+1_{j}\right)+D\left(x+1_{j}\right) \text {, by }(40) .
$$

Taking $y=x$, we obtain

$$
w\left(x+1_{j}\right) \geq w\left(0_{i}, x_{-i}\right)+D(x) .
$$

Hence, $w$ is a monotone multichoice game.

We now prove Proposition 3.

Proof of Proposition 3. Let $v \in \mathcal{G}(L)$, by Lemma 4 there exists a monotone multichoice game $w$, such that $\left|\Delta_{i} v(x)\right|=\left|\Delta_{i} w(x)\right|$ for all $x \in L, x_{i}<k_{i}$.

Observe that (CC) implies $\phi_{i}(\mathbf{0})=0$ for all $i \in N$, where $\mathbf{0}$ indicates the null game. Suppose now that $i$ is a null player in $w$. It follows that $\Delta_{i} w(x)=0=\Delta_{i} \mathbf{0}(x)$ for all $x$. Then by (MC), we deduce $\phi_{i}(w)=0$. In other words, $\phi$ satisfies the null axiom (N) for any game $w \in \mathcal{G}_{M}(L)$ (and even for any nonmonotonic game). Then proceeding as in the proof of Proposition 1, we deduce:

$$
\phi_{i}(w)=\sum_{\substack{x \in L \\ x_{i}<k_{i}}} p_{x}^{i}\left(w\left(x+1_{i}\right)-w(x)\right),
$$

and by axiom (MC) we have, for all $i \in N$,

$$
\begin{aligned}
\phi_{i}(v) & =\phi_{i}(w) \\
& =\sum_{\substack{x \in L \\
x_{i}<k_{i}}} p_{x}^{i}\left(w\left(x+1_{i}\right)-w(x)\right) \\
& =\sum_{\substack{x \in L \\
x_{i}<k_{i}}} p_{x}^{i}\left|\Delta_{i} v(x)\right| .
\end{aligned}
$$

The proof of Proposition 4 works as in the case of Proposition 1. 
Proof of Theorem 3. It is easy to check that the formula (23) satisfies the axioms (CC), (I), (SD), (AE) and (MC). Let us show that it satisfies the axiom (C). For every $x \in L$ such that $\sigma(x)=n$, we have,

$$
\begin{aligned}
\phi_{i}^{\mathrm{a}}\left(\delta_{x_{-i}, k_{i}}\right) & =\frac{\left(n-\sigma\left(x_{-i}\right)-1\right) ! \kappa\left(x_{-i}\right) !}{\left(n+\kappa\left(x_{-i}\right)-\sigma\left(x_{-i}\right)\right) !} \\
& =\frac{\kappa\left(x_{-i}\right) !}{\left(\kappa\left(x_{-i}\right)+1\right) !} \\
& =\frac{1}{\kappa(x)} .
\end{aligned}
$$

Conversely, we consider $\phi$ satisfying the axioms (CC), (I), (SD), (AE), (MC) and (C). Let $x \in L$, we write $x=\left(0_{N \backslash S \cup T}, x_{S}, k_{T}\right)$, with $x_{S} \in L_{S} \backslash \Gamma\left(L_{S}\right), S=\Sigma(x) \backslash \mathrm{K}(x)$, and $T=\mathrm{K}(x)$. By Proposition 4, we have

$$
\phi_{i}\left(\delta_{x}\right)=p_{x_{-i}}^{i} \sum_{\substack{y_{i} \in L_{i} \\ y_{i}<k_{i}}}\left|\delta_{x}\left(x_{-i}, y_{i}+1\right)-\delta_{x}\left(x_{-i}, y_{i}\right)\right|, \forall i \in N .
$$

Then we obtain,

$$
\phi_{i}\left(\delta_{\left(x_{-i}, k_{i}\right)}\right)=\phi_{i}\left(\delta_{\left(x_{-i}, 0_{i}\right)}\right)=p_{x_{-i}}^{i},
$$

and

$$
\phi_{i}\left(\delta_{\left(x_{-i}, x_{i}\right)}\right)=2 \phi_{i}\left(\delta_{\left(x_{-i}, k_{i}\right)}\right) \text {, for } x_{i} \in L_{i} \backslash\left\{0, k_{i}\right\} .
$$

We only have to determine $\phi_{i}\left(\delta_{\left(x_{-i}, k_{i}\right)}\right)$, with $x_{-i}=\left(0_{N \backslash S \cup T}, x_{S}, k_{T \backslash i}\right)$.

We distinguish the two following cases:

(i) If $S \cup T=N$, by axiom (C), we have

$$
\phi_{i}\left(\delta_{x_{N \backslash T}, k_{T \backslash i}, k_{i}}\right)=\frac{1}{t} .
$$

(ii) If $S \cup T \neq N$, by axioms (SD) and (AE), we have, $\forall t \in\{1, \ldots, n-1\}, \forall s \in\{1, \ldots, n-2\}$ with $s+t<n$,

$$
\phi_{i}\left(\delta_{\left(0_{N \backslash S \cup T}, x_{S}, k_{T \backslash i}, k_{i}\right)}\right)=\frac{n-s-t}{t} \phi_{j}\left(\delta_{\left(0_{j}, 0_{N \backslash S \cup T \cup j}, x_{S}, k_{T}\right)}\right),
$$

by (41), we obtain,

$$
\phi_{i}\left(\delta_{\left(0_{N \backslash S \cup T}, x_{S}, k_{T \backslash i}, k_{i}\right)}\right)=\frac{n-s-t}{t} \phi_{j}\left(\delta_{\left(0_{N \backslash S \cup T \cup j}, x_{S}, k_{T \cup j \backslash i}, k_{i}\right)}\right),
$$

and thus, recursively,

$$
\begin{aligned}
\phi_{i}\left(\delta_{\left(0_{N \backslash S \cup T}, x_{S}, k_{T \backslash i}, k_{i}\right)}\right) & =\frac{n-s-t}{t} \phi_{j}\left(\delta_{\left(0_{N \backslash S \cup T \cup j}, x_{S}, k_{T \cup\{j\}}\right)}\right) \\
& \vdots \\
& =\frac{(n-s-t) !(t-1) !}{(n-s-1) !} \phi_{j}\left(\delta_{\left(x_{S}, k_{N \backslash S \cup j}, k_{j}\right)}\right) .
\end{aligned}
$$


By axiom (C), we finally obtain

$$
\begin{aligned}
\phi_{i}\left(\delta_{\left(0_{N \backslash S \cup T}, x_{S}, k_{T \backslash i}, k_{i}\right)}\right) & =\frac{(n-s-t) !(t-1) !}{(n-s) !} \\
& =\frac{(n-s(x)) !(k(x)-1) !}{(n+k(x)-s(x)) !} \\
& =\frac{\left(n-s\left(x_{-i}\right)-1\right) !\left(k\left(x_{-i}\right)\right) !}{\left(n+k\left(x_{-i}\right)-s\left(x_{-i}\right)\right) !} .
\end{aligned}
$$

The result is proved.

Proof of Theorem 4. To keep notation simple we assume $k_{1}=\cdots=k_{n}=k$, but this is not limitative.

1. We first establish the following general result.

Lemma 5. Let $g: \mathbb{R}^{n} \rightarrow \mathbb{R}^{m}, m \leq n$, be a linear mapping, with $\operatorname{dim}(\operatorname{Ker}(g))=\ell$. Consider $f: \mathbb{R}^{n} \rightarrow \mathbb{R}^{m}$ a linear mapping. Then

$$
\operatorname{Ker}(f)=\operatorname{Ker}(g) \Leftrightarrow f=h \circ g,
$$

with $h: \mathbb{R}^{m} \rightarrow \mathbb{R}^{m}$ a linear mapping and $\operatorname{dim}(\operatorname{Ker}(h)) \leq m-n+\ell$.

Proof. Consider first that $\operatorname{Ker}(g)=\{0\}$, Then $n=m, \ell=0$ and $f, g, h$ are bijections, with $h=f \circ g^{-1}$.

Consider now that $\operatorname{Ker}(g) \neq\{0\}$, then $\ell \geq 1$. Choose $B=\left\{v_{1}, \ldots, v_{n}\right\}$ with $v_{i} \in \mathbb{R}^{m}$, a basis of $\mathbb{R}^{n}$ such that $\left\{v_{1}, \ldots, v_{\ell}\right\}$ is a basis of $\operatorname{Ker}(g)$. Then $g\left(v_{i}\right)=f\left(v_{i}\right)=0$ for $i=1, \ldots, \ell$, and $g, f$ are uniquely determined by the images of vectors $v_{l+1}, \ldots, v_{n}$ by $g$ and $f$. We claim that $g\left(v_{\ell+1}\right), \ldots, g\left(v_{n}\right)$ are independent vectors. Indeed, suppose there exist $\lambda_{\ell+1}, \ldots, \lambda_{n} \in \mathbb{R}$, not all equal to 0 , such that $\sum_{i=\ell+1}^{n} \lambda_{i} g\left(v_{i}\right)=0$. By linearity of $g$,

$$
0=\sum_{i=\ell+1}^{n} \lambda_{i} g\left(v_{i}\right)=g\left(\sum_{i=\ell+1}^{n} \lambda_{i} v_{i}\right),
$$

i.e., $\sum_{i=\ell+1}^{n} \lambda_{i} v_{i} \in \operatorname{Ker}(g)$, which is impossible since $\left\{v_{1}, \ldots, v_{\ell}\right\}$ is a basis of $\operatorname{Ker}(g)$. The same conclusion holds for $f\left(v_{\ell+1}\right), \ldots, f\left(v_{n}\right)$. Therefore, there exists a mapping $h: \mathbb{R}^{m} \rightarrow \mathbb{R}^{m}$ transforming the set of independent vectors $g\left(v_{\ell+1}\right), \ldots, g\left(v_{n}\right)$ into the set of independent vectors $f\left(v_{\ell+1}\right), \ldots, f\left(v_{n}\right)$, letting, e.g., $f\left(v_{i}\right)=h\left(g\left(v_{i}\right)\right), i=\ell+1, \ldots, n$, and $\operatorname{dim}(\operatorname{Ker}(h)) \leq m-(n-$ $\ell)$.

As a particular case, if $\operatorname{dim}(\operatorname{Ker}(g))=n-m$, then $h$ is a bijection.

2. Observe that the derivative of a game can be considered as a linear map $\Delta_{i}: \mathbb{R}^{(k+1)^{n}-1} \rightarrow$ $\mathbb{R}^{k(k+1)^{n-1}}$. Its kernel is the set of games $v$ such that $\Delta_{i} v=0$ pointwise (which is equivalent to: $i$ is null for $v$ ), i.e., $v\left(z+1_{i}\right)=v(z)$ for all $z \in L$ such that $z_{i}<k$, from which it follows that $v\left(z_{-i}, 0_{i}\right)=v\left(z_{-i}, 1_{i}\right)=\cdots=v\left(z_{-i}, k_{i}\right)$. Hence, $\operatorname{dim}\left(\operatorname{Ker}\left(\Delta_{i}\right)\right)=(k+1)^{n-1}-1$.

It is easy to see that the basis of the kernel is $\left\{u_{z}\right\}_{z \in L, z_{i}=0}$, therefore the basis of the supplementary space (let us call it $B$ ) is $\left\{u_{z}\right\}_{z \in L, z_{i}>0}$.

3. Define the linear map $g: \mathcal{G}(L) \rightarrow \mathcal{G}(L)$ by

$$
g\left(u_{z}\right)= \begin{cases}0, & \text { if } z \in L, z_{i}=0 \\ u_{z}, & \text { otherwise. }\end{cases}
$$


Then $\operatorname{Ker}(g)=\operatorname{Ker}\left(\Delta_{i}\right)$, and by Lemma 5 it follows that $g=h \circ \Delta_{i}$ and $h$ is a linear bijection on $\mathbb{R}^{k(k+1)^{n-1}}$. Moreover, $\operatorname{Im}(g)=B$.

Now, we can write $\phi_{i}=\psi \circ g$ with $\psi: B \rightarrow \mathbb{R}_{+}$, which defines $\psi$ unambiguously. We show that $\psi$ is a norm on $B$, which finishes the proof. Consider $v_{1}, v_{2} \in B$, then there exist $w_{1}, w_{2} \in \mathcal{G}(L)$ such that $v_{1}=g\left(w_{1}\right)$ and $v_{2}=g\left(w_{2}\right)$. We have:

- Suppose $\psi\left(v_{1}\right)=0$. Then $0=\psi\left(v_{1}\right)=\psi\left(g\left(w_{1}\right)\right)=\phi_{i}\left(w_{1}\right)$, hence by (SN) this is equivalent to $w_{1} \in \operatorname{Ker}\left(\Delta_{i}\right)=\operatorname{Ker}(g)$, equivalent to $v_{1}=g\left(w_{1}\right)=0$.

- For all $\alpha \in \mathbb{R}, \psi\left(\alpha v_{1}\right)=\psi\left(\alpha g\left(w_{1}\right)\right)=\psi\left(g\left(\alpha w_{1}\right)\right)=\phi_{i}\left(\alpha w_{1}\right)$, which by (AH) is equal to $|\alpha| \phi_{i}\left(w_{1}\right)=|\alpha| \psi\left(v_{1}\right)$.

- $\psi\left(v_{1}+v_{2}\right)=\psi\left(g\left(w_{1}+w_{2}\right)\right)=\phi_{i}\left(w_{1}+w_{2}\right) \leq \phi_{i}\left(w_{1}\right)+\phi_{i}\left(w_{2}\right)=\psi\left(v_{1}\right)+\psi\left(v_{2}\right)$, where we have used (SA).

Hence, $\psi$ is a norm on $B$.

Proof of Theorem 5. Let $v$ a $k_{N}$-ary capacity. For every $i \in N$, we have, by Lemma 3 ,

$$
\begin{aligned}
\phi_{i}^{\mathrm{s}}(v)= & \sum_{\substack{x \in L \\
\forall j \in N, x_{j}<k_{j}}} \phi_{i}^{S h}\left(\mu_{x}^{v}\right) \\
= & \sum_{\substack{x \in L \\
\forall j \in N, x_{j}<k_{j}}} \int_{[0,1]^{n}} \frac{\partial C_{\mu_{x}^{v}}(t)}{\partial t_{i}} d t \\
= & \sum_{\substack{x \in L \\
\forall j \in N, x_{j}<k_{j}}} \int_{\left[x, x+1_{N}\right]} \frac{\partial C_{\mu_{x}^{v}}(z-x)}{\partial z_{i}} d z \\
= & \sum_{\substack{x \in L \\
\forall j \in N, x_{j}<k_{j}}} \int_{\left[x, x+1_{N}\right]} \frac{\partial \mathcal{C}_{v}(z)}{\partial z_{i}} d z \\
= & \int_{\left[0, k_{N}\right]} \frac{\partial \mathcal{C}_{v}(z)}{\partial z_{i}} d z .
\end{aligned}
$$

Hence (29) is proved.

Let $v \in \mathcal{G}(L)$, by Lemma 4 there exists $w \in \mathcal{G}_{M}(L)$ such that $\left|\Delta_{i} v(x)\right|=\left|\Delta_{i} w(x)\right|$ for all $x \in L, x_{i}<k_{i}$. Then $\left|\frac{\partial \mathcal{C}_{v}(x)}{\partial x_{i}}\right|=\left|\frac{\partial \mathcal{C}_{w}(x)}{\partial x_{i}}\right|$ for all $x \in\left[0, k_{N}\right]$. Hence, $\psi_{i}^{\mathrm{a}}\left(\mathcal{C}_{v}\right)=\psi_{i}^{\mathrm{a}}\left(\mathcal{C}_{w}\right)=\psi_{i}^{\mathrm{s}}\left(\mathcal{C}_{w}\right)$, and by (29), we have

$$
\psi_{i}^{\mathrm{a}}\left(\mathcal{C}_{v}\right)=\phi_{i}^{\mathrm{s}}\left(\mathcal{C}_{w}\right) .
$$


By Theorem 1 we have

$$
\begin{aligned}
\phi_{i}^{\mathrm{s}}\left(\mathcal{C}_{w}\right) & =\sum_{x_{-i} \in L_{-i}} \frac{\left(n-\sigma\left(x_{-i}\right)-1\right) ! \kappa\left(x_{-i}\right) !}{\left(n+\kappa\left(x_{-i}\right)-\sigma\left(x_{-i}\right)\right) !}\left(w\left(x_{-i}, k_{i}\right)-w\left(x_{-i}, 0_{i}\right)\right) \\
& =\sum_{x_{-i} \in L_{-i}} \frac{\left(n-\sigma\left(x_{-i}\right)-1\right) ! \kappa\left(x_{-i}\right) !}{\left(n+\kappa\left(x_{-i}\right)-\sigma\left(x_{-i}\right)\right) !} \sum_{\substack{x_{i} \in L_{i} \\
x_{i}<k_{i}}}\left|w\left(x+1_{i}\right)-w(x)\right| \\
& =\sum_{\substack{x \in L \\
x_{i}<k_{i}}} \frac{\left(n-\sigma\left(x_{-i}\right)-1\right) ! \kappa\left(x_{-i}\right) !}{\left(n+\kappa\left(x_{-i}\right)-\sigma\left(x_{-i}\right)\right) !}\left|v\left(x+1_{i}\right)-v(x)\right| \\
& =\phi_{i}^{\mathrm{a}}\left(\mathcal{C}_{v}\right) .
\end{aligned}
$$

Hence (30) is proved.

Proof of Theorem 6. It is easy to check that the formula satisfies all axioms.

Conversely, assume that $\phi^{m, M}$ satisfies all axioms, we have $\phi^{0_{N}, 1_{N}}=\phi^{S h}$.

From (32) and (T), we have

$$
\begin{aligned}
& \phi_{i}^{m, M}(v)=\sum_{y \in L^{m, M-1_{N}}} \phi_{i}^{0_{N}, 1_{N}}\left(T_{y} \circ v_{\mid L^{y, y+1_{N}}}\right) \\
& =\sum_{y \in L^{m, M-1} N} \phi_{i}^{S h}\left(T_{y} \circ v_{\mid L^{y, y+1} N}\right) \\
& =\sum_{y \in L^{m, M-1} 1_{N}} \sum_{x_{-i} \in L_{-i}^{0_{N}, 1_{N}}} \frac{\left(n-\sigma\left(x_{-i}\right)-1\right) ! \sigma\left(x_{-i}\right) !}{n}\left(T_{y} \circ v_{\mid L^{y, y+1_{N}}}\left(x+1_{i}\right)-T_{y} \circ v_{\mid L^{y, y+1} N}(x)\right) \\
& =\sum_{y \in L^{m, M-1} N_{N}} \sum_{x_{-i} \in L_{-i}^{0_{N}, 1_{N}}} \frac{\left(n-\sigma\left(x_{-i}\right)-1\right) ! \sigma\left(x_{-i}\right) !}{n}\left(v\left(x+y+1_{i}\right)-v(x+y)\right) \\
& =\sum_{y \in L^{m, M-1} N} \sum_{S \subseteq N \backslash i} \frac{(n-s-1) ! s !}{n}\left(v\left(y+1_{S \cup i}\right)-v\left(y+1_{S}\right)\right) \\
& =\sum_{\substack{z \in L^{m, M} \\
z_{i}<M_{i}}}\left(v\left(z+1_{i}\right)-v(z)\right) \sum_{\substack{S \subseteq N \backslash i \\
\forall j \in \overline{S, z_{j}>m_{j}} \\
\forall j \in N \backslash S, z_{j}<M_{j}}} \frac{(n-s-1) ! s !}{n !} \\
& =\sum_{\substack{z \in L^{m, M} \\
z_{i}<M_{i}}}\left(v\left(z+1_{i}\right)-v(z)\right) \sum_{\substack{S \subseteq(N \backslash i) \cap \sum\left(z_{-i}\right) \\
S \supseteq \mathrm{K}\left(z_{-i}\right)}} \frac{(n-s-1) ! s !}{n !} \\
& =\sum_{\substack{z \in L^{m, M} \\
z_{i}<M_{i}}}\left(v\left(z+1_{i}\right)-v(z)\right) \sum_{\substack{S \subseteq \sum_{S}\left(z_{-i}\right) \\
S \supseteq \mathrm{K}\left(z_{-i}\right)}} \frac{(n-s-1) ! s !}{n !} \\
& =\sum_{\substack{z \in L_{i}^{m, M} \\
z_{i}<M_{i}}} \frac{\left(n-\sigma\left(z_{-i}\right)-1\right) ! \kappa\left(z_{-i}\right) !}{\left(n+\kappa\left(z_{-i}\right)-\sigma\left(z_{-i}\right)\right) !}\left(v\left(z+1_{i}\right)-v(z)\right) .
\end{aligned}
$$

Proof of Proposition 5. It is easy to check that the formula satisfies all axioms. 
Conversely, assume that $\phi^{m, M}$ satisfies all axioms. For every $v \in \mathcal{G}_{M}\left(L^{0_{N}, 1_{N}}\right)$, we have $\phi^{0_{N}, 1_{N}}(v)=\phi^{S h}(v)$. Let now $v \in \mathcal{G}\left(L^{m, M}\right)$, by Lemma 4 there exists a multichoice game $w \in \mathcal{G}_{M}\left(L^{m, M}\right)$ such that $\left|\Delta_{i} v(x)\right|=\left|\Delta_{i} w(x)\right|$ for all $x \in L^{m, M}, x_{i}<M_{i}$. From (32) and (T), we have

$$
\begin{aligned}
\phi_{i}^{m, M}(w) & =\sum_{y \in L^{m, M-1} N} \phi_{i}^{0_{N}, 1_{N}}\left(T_{y} \circ w_{\mid L^{y, y+1} N}\right) \\
& =\sum_{y \in L^{m, M-1} 1_{N}} \phi_{i}^{S h}\left(T_{y} \circ w_{\mid L^{y, y+1_{N}}}\right) \\
& =\sum_{\substack{x \in L^{m, M} \\
x_{i}<M_{i}}} \frac{\left(n-\sigma\left(x_{-i}\right)-1\right) ! \kappa\left(x_{-i}\right) !}{\left(n+\kappa\left(x_{-i}\right)-\sigma\left(x_{-i}\right)\right) !}\left(w\left(x+1_{i}\right)-w(x)\right) .
\end{aligned}
$$

The result is then obvious by (MC).

\section{Appendix B Logical independence of Axioms for Theorem 2}

The following indices show that the four axioms (L), (I'), (S D) and (E) are logically independent.

- Consider the value $\phi$ defined by

$$
\forall v \in \mathcal{G}(L), \forall i \in N, \phi_{i}(v)=0 .
$$

This value satisfies (L), (I'), (S D) but not $(\mathbf{E})$.

- Consider the value $\phi$ given by

$$
\forall v \in \mathcal{G}(L), \forall i \in N, \phi_{i}(v)=\sum_{\substack{x \in L \\ \forall j \in N, x_{j}<k_{j}}}\left(\frac{\mu_{x}^{v}(N)}{n}+\sum_{\substack{S \neq N \\ S \ni i}}(n-s)\left(s \mu_{x}^{v}(S)-(n-s) \mu_{x}^{v}(N \backslash S)\right)\right),
$$

with $\mu_{x}^{v}(S)=v\left(x+1_{S}\right)-v(x), \forall S \subseteq N$. This value satisfies (L), (SD) and (E). However, it does not satisfy the invariance axiom (I').

- The value $\phi$ on $\mathcal{G}(L)$ defined for each $v \in \mathcal{G}(L)$ and each $i \in N$, by

$$
\phi_{i}(v)=\sum_{\substack{x \in L \\ \forall j \in N, x_{j}<k_{j}}} \frac{\beta_{i}\left(\mu_{x}^{v}\right)}{\sum_{i \in N} \beta_{i}\left(\mu_{x}^{v}\right)} \mu_{x}(N),
$$

where $\beta_{i}$ is the Banzhaf value on $\mathcal{G}\left(2^{N}\right)$ and $\mu_{x}^{v}(A)=v\left(x+1_{A}\right)-v(x), \forall A \subseteq N$, satisfies $\left(\mathbf{I}^{\prime}\right),(\mathrm{SD}),(\mathbf{E})$ but not (L).

- We consider the value $\phi$ defined by

$$
\forall v \in \mathcal{G}(L), \forall i \in N, \phi_{i}(v)=\sum_{\substack{x \in L \\ \forall j \in N, x_{j}<k_{j}}}\left(v\left(x+1_{\{i, \ldots, n\}}\right)-v\left(x+1_{\{i+1, \ldots, n\}}\right)\right) .
$$

The value $\phi$ satisfies (L), (I'), (E) but not (S D). 


\section{Appendix C Logical independence of Axioms for Theorem 3}

- Consider the value $\phi$ defined by

$$
\forall v \in \mathcal{G}(L), \forall i \in N, \phi_{i}(v)=0 .
$$

This value satisfies (CC), (I), (SD), (AE), (MC) but not (C).

- Consider the value $\phi$ given by

$$
\forall v \in \mathcal{G}(L), \forall i \in N, \phi_{i}(v)=\phi_{i}^{\mathrm{a}}(v)+\sum_{\substack{x \in L \backslash\left\{0_{N}, k_{N}\right\} \\ x_{i}<k_{i}-1}}\left|v\left(x+1_{i}\right)-v(x)\right| .
$$

This value satisfies (CC), (SD), (MC), (AE) and (C). However, it does not satisfy the invariance axiom (I).

- We consider the value $\phi$ defined by

$$
\forall v \in \mathcal{G}(L), \forall i \in N, \phi_{i}(v)= \begin{cases}\phi_{i}^{\mathrm{a}}(v), & \text { if } i \in S(v) \\ \sum_{x \in L, x_{i}<k_{i}}\left|v\left(x+1_{i}\right)-v(x)\right|, & \text { otherwise }\end{cases}
$$

with $S(v)=\bigcup_{x \in L: v(x) \neq 0} \Sigma(x)$.

The value $\phi$ satisfies (CC), (SD), (MC), (I), (C) but not (AE).

- Consider the value $\phi$ given by

$$
\forall v \in \mathcal{G}(L), \forall i \in N, \phi_{i}(v)= \begin{cases}\phi_{i}^{\mathrm{a}}(v), & \text { if } i \in S(v) \backslash K(v) \\ \phi_{i}^{\mathrm{s}}(v), & \text { if } i \in K(v) \\ -\phi_{i}^{\mathrm{s}}(v), & \text { otherwise }\end{cases}
$$

with $S(v)=\bigcup_{x \in L: v(x) \neq 0} \Sigma(x)$, and $K(v)=\bigcup_{x \in L: v(x) \neq 0} \kappa(x)$

This value satisfies (CC), (SD), (I), (AE) and (C). However, it does not satisfy the marginal contribution axiom (MC).

- The value $\phi$ on $\mathcal{G}(L)$ defined for each $v \in \mathcal{G}(L)$ and each $i \in N$, by

$$
\phi_{i}(v)=\sum_{\substack{x \in L \\ \forall j \in N, x_{j}<k_{j}}}\left|\left(v\left(x+1_{\{i, \ldots, n\}}\right)-v\left(x+1_{\{i+1, \ldots, n\}}\right)\right)\right|,
$$

satisfies (CC), (I), (AE), (MC) and (C) but not (S D).

- We consider the value $\phi$ defined by

$$
\forall v \in \mathcal{G}(L), \forall i \in N, \phi_{i}(v)=\phi_{i}^{\mathrm{a}}(v)+1 .
$$

The value $\phi$ satisfies (SD), (MC), (I), (C) and (AE) but not (CC). 


\section{References}

J. Banzhaf. Weighted voting doesn't work: A mathematical analysis. Rutgers Law Review, 19: 317-343, 1965.

G. Choquet. Theory of capacities. Annales de l'institut Fourier, 5:131-295, 1953.

Y. Crama and P. Hammer. Boolean functions. Number 142 in Encyclopedia of Mathematics and Its Applications. Cambridge University Press, 2011.

A. Datta, S. Sen, and Y. Zick. Algorithmic transparency via quantitative input influence: Theory and experiments with learning systems. In 2016 IEEE Symposium on Security and Privacy (SP), pages 598-617.

A. Denguir. Modèle de performance agrégée et raisonnement approché pour l'optimisation de la consommation énergétique et du confort dans les bâtiments. PhD thesis, Montpellier 2, 2014.

P. Fanger. Thermal comfort: Analysis and applications in environmental engineering. Copenhagen: Danish Technical Press, 1970.

P. Fishburn. Interdependence and additivity in multivariate, unidimensional expected utility theory. International Economic Review, 8:335-342, 1967.

M. Grabisch. The application of fuzzy integrals in multicriteria decision making. European Journal of Operational Research, 89:445-456, 1996.

M. Grabisch and Ch. Labreuche. Capacities on lattices and $k$-ary capacities. In International Conference of the Euro Society for Fuzzy Logic and Technology (EUSFLAT), pages 304-307, Zittau, Germany, 2003.

M. Grabisch and Ch. Labreuche. Bipolarization of posets and natural interpolation. Journal of Mathematical Analysis and Application, 343:1080-1097, 2008.

M. Grabisch and Ch. Labreuche. A decade of application of the Choquet and Sugeno integrals in multi-criteria decision aid. Annals of Operations Research, 175:247-286, 2010.

M. Grabisch and Ch. Labreuche. Fuzzy measures and integrals in MCDA. In S. Greco, M. Ehrgott, and J.R. Figueira, editors, Multiple Criteria Decision Analysis, pages 553-603. Springer, 2016.

M. Grabisch and Ch. Labreuche. Monotone decomposition of 2-additive generalized additive independence models. Mathematical Social Sciences, 92:64-73, 2018.

M. Grabisch and F. Lange. Games on lattices, multichoice games and the Shapley value: a new approach. Mathematical Methods of Operations Research, 65(1):153-167, 2007.

M. Grabisch, J.-L. Marichal, and M. Roubens. Equivalent representations of set functions. Mathematics of Operations Research, 25(2):157-178, 2000.

M. Grabisch, J.-L. Marichal, R. Mesiar, and E. Pap. Aggregation Functions. Cambridge University Press, Encyclopedia of Mathematics and its Applications: Vol. 127, 2009.

F. Haldi and D. Robinson. Modelling occupants personal characteristics for thermal comfort prediction. International Journal of Biometeorology, 55(5):681-694, 2011. 
P.L. Hammer and S. Rudeanu. Boolean methods in operations research and related areas, volume 7 of Econometrics and Operations Research. Springer, 1968.

C. R. Hsiao and T. E. S. Raghavan. Shapley value for multi-choice cooperative games, I. Games and Economic Behavior, 5:240-256, 1993.

F. Klijn, M. Slikker, and J.M. Zarzuelo. Characterizations of a multi-choice value. International Journal of Game Theory, 28:521-532, 1999.

Ch. Labreuche and S. Fossier. Explaining multi-criteria decision aiding models with an extended Shapley value. In International Joint Conference on Artificial Intelligence (IJCAI'2018), pages 331-339, 2018.

Ch. Labreuche and M. Grabisch. Using multiple reference levels in Multi-Criteria Decision Aid: the Generalized-Additive Independence model and the Choquet integral approaches. European Journal of Operational Research, 267(2):598 - 611, 2018.

S. Lundberg and S.I. Lee. A unified approach to interpreting model predictions. In 31st Conference on Neural Information Processing Systems (NIPS 2017), pages 4765-4774, Long Beach, CA, USA., 2017. Curran Associates, Inc.

J.-L. Marichal. Aggregation operators for multicriteria decision aid. $\mathrm{PhD}$ thesis, University of Liège, 1998.

T. Murofushi. A technique for reading fuzzy measures (I): the Shapley value with respect to a fuzzy measure. In 2nd Fuzzy Workshop, pages 39-48, Nagaoka, Japan, October 1992. In Japanese.

G. Owen. Multilinear extensions of games. Management Science, 18(5):64-79, 1972.

B. Peleg and P. Sudhölter. Introduction to the Theory of Cooperative Games. Kluwer Academic Publisher, 2003.

H. Peters. Game Theory: A Multilevel Approach. Springer, 2008.

H. Peters and H. Zank. The egalitarian solution for multichoice games. Annals of Operations Research, 137:399-409, 2005.

M. Ridaoui, M. Grabisch, and Ch. Labreuche. An alternative view of importance indices for multichoice games. In Algorithmic Decision Theory, pages 81-92, 2017a.

M. Ridaoui, M. Grabisch, and Ch. Labreuche. Axiomatization of an importance index for generalized additive independence models. In 14th European Conference, ECSQARU 2017, Lugano, Switzerland, July 2017, pages 340-350, 2017b.

G. C. Rota. On the foundations of combinatorial theory I. Theory of Möbius functions. Zeitschrift für Wahrscheinlichkeitstheorie und Verwandte Gebiete, 2(4):340-368, 1964.

L. S. Shapley. A value for n-person games. In H. W. Kuhn and A. W. Tucker, editors, Contributions to the Theory of Games, Vol. II, number 28 in Annals of Mathematics Studies, pages 307-317. Princeton University Press, 1953.

A. van den Nouweland, S. Tijs, J. Potters, and J. Zarzuelo. Cores and related solution concepts for multi-choice games. Zeitschrift für Operations Research, 41:289-311, 1995. 